\title{
Topological Sigma Models
}

\author{
Edward Witten* \\ School of Natural Sciences, Institute for Advanced Study, Olden Lane, Princeton, NJ 08540, USA
}

\begin{abstract}
A variant of the usual supersymmetric nonlinear sigma model is described, governing maps from a Riemann surface $\Sigma$ to an arbitrary almost complex manifold $M$. It possesses a fermionic BRST-like symmetry, conserved for arbitrary $\Sigma$, and obeying $Q^{2}=0$. In a suitable version, the quantum ground states are the $1+1$ dimensional Floer groups. The correlation functions of the BRST-invariant operators are invariants (depending only on the homotopy type of the almost complex structure of $M$ ) similar to those that have entered in recent work of Gromov on symplectic geometry. The model can be coupled to dynamical gravitational or gauge fields while preserving the fermionic symmetry; some observations by Atiyah suggest that the latter coupling may be related to the Jones polynomial of knot theory. From the point of view of string theory, the main novelty of this type of sigma model is that the graviton vertex operator is a BRST commutator. Thus, models of this type may correspond to a realization at the level of string theory of an unbroken phase of quantum gravity.
\end{abstract}

\section{Introduction}

In recent years, Yang-Mills instantons have played an important role in the study of four manifolds and three manifolds in the work of Donaldson [1] and Floer [2], respectively. More recently, Atiyah advocated an interpretation of Floer theory in terms of a non-relativistic version of supersymmetric quantum Yang-Mills theory [3] and offered some evidence that this might have a relativistic generalization that would account for many features of Donaldson and Floer theory. A relativistic quantum field theory that seems to have the requisite properties was indeed formulated in [4]. It possesses a global fermionic symmetry which is similar in many ways to BRST symmetry, though it can be obtained by twisting ordinary $N=2$ supersymmetric Yang-Mills theory.

There is also a $1+1$ dimensional version of Floer theory [2], which has given striking results about symplectic diffeomorphisms of symplectic manifolds. From

\footnotetext{
* On leave from Department of Physics, Princeton University. Supported in part by NSF Grants No. 80-19754, 86-16129, 86-20266
} 
the considerations in [3], it is evident that $1+1$ dimensional Floer theory is related to some version of supersymmetric nonlinear sigma models. In the present paper, we will attempt to clarify this and find the precise version of relativistic sigma models that enters.

There are three motivations for the present work. First of all, the problem just stated of understanding the relativistic generalization of $1+1$ dimensional Floer theory is clearly interesting in itself. In fact, we will find an interesting variant of the usual supersymmetric nonlinear sigma model with a BRST-like fermionic symmetry. The correlation functions of the BRST invariant operators that appear are invariants, similar to those in work of Gromov [5] on symplectic geometry.

Second, Atiyah has also conjectured [3] that the Jones knot polynomial [6] should have a natural description in terms of Floer and Donaldson theory. It is tempting to think that, if so, $1+1$ dimensional as well as $3+1$ dimensional Floer theory should play a role. A knot is after all an embedding $\phi: S \rightarrow Y$, where $S$ is a circle and $Y$ a three manifold. (One sometimes specializes to the case that $Y$ is a three-sphere, but we do not wish to do this.) What could be more natural than to consider on $S$ a nonlinear sigma model with BRST-like symmetry, coupled to gauge fields on $Y$ in a BRST-invariant fashion? In essence, one is thus considering the knot as a "superconducting cosmic knot," with current-carrying modes that are coupled to gauge fields in three-space. ${ }^{1}$ The BRST-invariant correlation functions are in principle then knot invariants, perhaps related to the Jones polynomial, though we will have to leave this question for the future.

The final motivation for the present work is connected with the possible physical implications of Donaldson and Floer theory. The fermionic symmetry in [4] is formally very similar to BRST symmetry. For instance, the fermionic charge is a Lorentz scalar $Q$ obeying $Q^{2}=0$. It is very possible that this fermionic symmetry is in fact a BRST symmetry that arises in gauge fixing of some underlying system with higher gauge invariance. For reasons sketched in [4], if such an underlying theory exists it must be a generally covariant theory in an exotic phase in which general covariance is unbroken; as a result, signals cannot propagate, and the only observables are global topological invariants, the Donaldson polynomials. It does not seem likely that there is any ordinary quantum field theory in which the fermionic symmetry of [4] (or its gravitational analogue [10]) arises by BRST gauge fixing. A plausible higher symmetry that could do the job upon gauge fixing is not known in field theory.

But it is very intriguing to ask whether string theory, which certainly does possess higher gauge invariances of a mysterious kind, is the right framework in which such fermionic symmetries arise naturally as BRST symmetries after gauge fixing. It is almost always logical to try to understand a symmetry by considering a situation in which the symmetry is unbroken. In the case of string theory, there is a particularly compelling reason for this. As long as general covariance is broken by a choice of metric tensor, the metric plays a key role in the propagation of

\footnotetext{
${ }^{1}$ There is a striking analogy of such a system with superconducting cosmic strings [7] and also with the incorporation of current carrying modes on fundamental strings [8]. There is an interesting analogy, made explicit in [9], between the two types of current-carrying string
} 
signals of all kinds, including strings. In string theory, such a distinguished role for the metric tensor in unnatural, the metric being just one of infinitely many string modes. To properly understand the higher symmetries of string theory, one would like to put the graviton on the same basis as all the other modes, and perhaps this is most natural in a phase in which general covariance is unbroken.

What would the unbroken phase look like in string theory? One possibility which has been proposed [11] (a review can be found in [12]) involves string field theory. Another interesting suggestion involves trying to infer properties of the unbroken phase from the behavior of scattering amplitudes at high energies and fixed angles [13]. On the other hand, it is tempting to ask whether the unbroken phase of string theory, like the broken phase, might have a representation in terms of world sheet path integrals. ${ }^{2}$ In the spirit of $[4,10]$, it is pretty clear how one should try to find the unbroken phase in world sheet path integrals. One should look for nonlinear sigma models with BRST-like fermionic symmetry and with the property that the graviton vertex operator is a BRST commutator. This is what we will do in this paper, in the process of understanding $1+1$ dimensional Floer theory. Thus it is tempting to propose that the models considered in this paper correspond to the unbroken phase of string theory. This must, however, be regarded as a rather tentative conjecture for a whole host of reasons, among them the fact that we will be considering manifolds with almost complex structure, something that is not part of our usual thinking about space-time. ${ }^{3}$

The organization of this paper is a follows. In Sect. 2, we describe the relativistic sigma models which seem to be related to $1+1$ dimensional Floer theory. In Sect. 3, we determine the BRST invariant vertex operators and sketch the relation to Gromov's work. In Sect. 4, we couple the model to dynamical two dimensional gravity (after introducing the two dimensional analogue of "topological gravity"). In Sect. 5, we couple to dynamical gauge fields, and define some knot invariants which may or may not prove to be interest.

\section{Construction of the Lagrangian}

The two dimensional nonlinear sigma model is a theory of maps from a Riemann surface $\Sigma$ to a Riemannian manifold $M$. The usual supersymmetric nonlinear sigma model possess fermionic symmetries whose anticommutators generate infinitesimal bosonic symmetries of $\Sigma$. Such infinitesimal bosonic symmetries

\footnotetext{
2 Like the world sheet path integral description of the broken phase, this description of the unbroken phase, if it can be formulated, may well need a more fundamental formulation. World sheet path integrals should probably be seen as a structure which arises upon trying to expand in perturbation theory some underlying (and presently unknown) geometrical structure

${ }^{3}$ Notice, though, that the cotangent bundle of space-time (or any manifold $M$ ) has a canonical symplectic structure, which, with a choice of metric on space-time, automatically gives also an almost complex structure. Perhaps this is the proper context for relating the models considered in this paper to string theory. Twistor theory suggests some other ways to associate an almost complex manifold to any given real manifold $M$. For instance, one can consider the bundle over $M$ whose fiber at a point $x \in M$ is the space of all almost complex structures on the tangent space to $M$ at $x$. The total space $\hat{M}$ of this bundle has (with a choice of metric on $M$ ) a natural almost complex structure
} 
correspond to global holomorphic vector fields, and so exist only if $\Sigma$ has genus zero or one. In this section, we will formulate a variant of the usual supersymmetric nonlinear sigma model which possesses a global fermionic symmetry on a Riemann surface $\Sigma$ of any genus. This is possible because the fermionic charge $Q$ obeys $Q^{2}=0$ (rather than $Q^{2}$ generating an infinitesimal bosonic symmetry, as in the usual case).

In the usual supersymmetric nonlinear sigma model, the fermionic symmetries have spin $+\frac{1}{2}$ or spin $-\frac{1}{2}$, and act on right or left moving modes. The non-zero spin carried by the fermionic charges makes it possible in the usual situation to decompose any given fermionic charge into left or right handed eigencharges of definite spin. In the situation that we will consider, there will be just one fermionic charge $Q$, with spin zero so that it has no decomposition into components of positive and negative spin. $Q$ will couple to both left and right moving modes, in such way that (just as in [4]) every field has a $Q$-partner. As a result, the $Q$ cohomology groups (solutions of $Q \chi=0$, modulo the equivalence relation $\chi \simeq \chi+Q \lambda$ ) are purely global invariants of $M$, the Floer groups; there are no local degrees of freedom in the BRST sense. As was indicated in the introduction, this means that the sigma models we will consider correspond roughly to an unbroken phase of string theory.

In the work of Floer and Gromov, it is important that $M$ should have an almost complex structure. We will accept this as a cue and attempt to formulate a sigma model that depends on such an almost complex structure. (To make closer contact with the results of Floer and Gromov, we need an additional structure on $M-$ a symplectic structure. From our viewpoint, this enters not in the basic construction of the Lagrangian but in extracting some of its geometrical consequences, as we will see in Sect. 3.)

Let us recall the definition of an almost complex structure. Let $T$ denote the tangent bundle of $M$. The linear transformations of $T$ correspond to a vector bundle End $T{ }^{4}$ An almost complex structure is a section $J$ of End $T$ such that $J^{2}=-1$. Upon picking a local trivialization of $T$, a vector field $V$ can be described by its components $V^{i}, i=1 \ldots n$ (here $n=\operatorname{dim} M$ ). The almost complex structure then corresponds to a tensor field $J^{i}{ }_{j}$ obeying

$$
J_{j}^{i} J^{j}{ }_{k}=-\delta^{i}{ }_{k} \text {. }
$$

On $M$, we consider a Riemannian metric $g_{i j}$ of type $(1,1)$. The statement that $g$ is of type $(1,1)$ means that

$$
g_{i j}=J^{s} J^{t}{ }_{j} g_{s t}
$$

or equivalently that

$$
J_{i l}=-J_{l i}
$$

where $J_{i l}=g_{i s} J^{s}$.

As a Riemannian manifold, $M$ possesses its Levi-Civita connection--the unique torsion free connection such that $D_{k} g_{i j}=0$. We will not assume that $D_{k} J^{i}{ }_{j}$

${ }^{4}$ That is, the fiber of End $T$ at a point $P \in M$ is the space of linear transformations of the fiber of $T$ at $P$ 
vanishes. The case $D_{k} J^{i}{ }_{j}=0$ is precisely the case in which $J$ is an integrable complex structure and $g$ is a Kahler metric. In that case, the nonlinear sigma model that we will construct simplifies considerably and reduces to a twisted version of the usual $N=2$ supersymmetric sigma model.

A map $\phi: \Sigma \rightarrow M$ can (locally) be described by functions $u^{i}(\sigma)(\sigma$ denotes a point in $\Sigma$, and the $u^{i}$ correspond to coordinates on $M$ ). In the nonlinear sigma model, these correspond to fields $u^{i}$ of conformal spin zero. The other fields in our construction will be the following. We introduce an anticommuting field $\chi^{i}(\sigma)$, a section of $\phi^{*}(T)$, the pullback of the tangent bundle of $T$ to $M$. It will have conformal spin zero. And we require an anticommuting field $\rho_{\beta}{ }^{i}(\sigma)$, of conformal spin one. $\left(\beta=1,2\right.$ is a tangent index to $\Sigma$, and $i=1 \ldots n$ runs over a basis of $\phi^{*}(T)$,) $\rho$ is a one form on $\Sigma$ with values in $\phi^{*}(T) \cdot \rho_{\beta}{ }^{i}$ obeys a "self-duality" constraint

$$
\rho^{\alpha i}=\varepsilon_{\beta}^{\alpha} J_{j}^{i} \rho^{\beta j} .
$$

Here $\varepsilon_{\beta}^{\alpha}$ is the complex structure of $\Sigma$, obeying $\varepsilon_{\beta}^{\alpha} \varepsilon^{\beta}{ }_{\gamma}=-\delta^{\alpha}{ }_{\gamma}$. Indices tangent to $\Sigma$ are raised and lowered by use of a metric $h_{\alpha \beta}$ on $\Sigma$, so $\rho^{\alpha i}=h^{\alpha \beta} \rho_{\beta}{ }^{i}, \varepsilon_{\alpha \beta}=h_{\alpha \gamma} \varepsilon_{\beta}{ }_{\beta}$, etc. $h_{\alpha \beta}$ is compatible with the complex structure $\varepsilon_{\beta}^{\alpha}$ in the sense that $h$ is of type $(1,1)$, so that $\varepsilon_{\alpha \beta}=-\varepsilon_{\beta \alpha}$. Finally, we introduce a commuting field $H^{\alpha i}$, a section of the same bundle as $\rho^{\alpha i}$, and obeying the same constraint

$$
H^{\alpha i}=\varepsilon_{\beta}^{\alpha} J_{j}^{i} H^{\beta j} \text {. }
$$

It will turn out that $H^{\alpha i}$ is not a propagating field; that is, the interesting Lagrangians will be such that the Euler-Lagrange equations enable one to solve for $H$ algebraically in terms of the other fields. It would be possible to write invariant Lagrangians without introducing $H$, but the importance of $H$ is that its introduction makes it possible to "close the algebra" (that is, to ensure $Q^{2}=0$ ) without using the equations of motion.

In addition to conformal invariance, and the fermionic symmetry $Q$, the model that we will construct will have at the classical level a bosonic symmetry $U$ which corresponds in Floer theory to the grading of the Floer complex and so obeys $[U, Q]=Q$. The fields $u, \lambda, \rho$, and $H$ have $U=0,1,-1$ and 0 , respectively. Their conformal dimensions are $0,0,1$ and 1 . This is summarized in the table.

Before attempting to construct a Lagrangian, let us state a few more conventions. The standard (torsion-free, metric compatible) affine connection of $M$ will be denoted $\Gamma_{j k}^{i}$. The covariant derivative of a vector is thus $D_{k} V^{i}=$ $\partial_{k} V^{i}+\Gamma_{k l}^{i} V^{l}$. The Riemann tensor is $R_{j k l}^{i}=\partial_{j} \Gamma_{k l}^{i}-\partial_{k} \Gamma_{j l}^{i}+\Gamma_{j s}^{i} \Gamma_{k l}^{s}-\Gamma_{k s}^{i} \Gamma_{j l}^{s}$.

Table I. The conformal dimensions, global charges, and statistics of the various fields

\begin{tabular}{llrl}
\hline & $D$ & $U$ & Statistics \\
\hline$u^{i}$ & 0 & 0 & + \\
$\chi^{i}$ & 0 & 1 & - \\
$\rho^{\alpha}$ & 1 & -1 & - \\
$H^{\beta i}$ & 1 & 0 & + \\
\hline
\end{tabular}


This definition is such that $\left[D_{j}, D_{k}\right] V^{i}=R_{j k}{ }_{l}^{i} V^{l}$. A connection on $\phi^{*}(T)$ is obtained by pulling back the connection of $T$ from $M$ to $\Sigma$, giving the covariant derivative of $\chi^{i}$,

$$
D_{\alpha} \chi^{i}=\partial_{\alpha} \chi^{i}+\partial_{\alpha} u^{k} \Gamma_{k l}^{i} \chi^{l},
$$

and similarly for any other section of $\phi^{*}(T)$.

We first postulate the fermionic transformation laws

$$
\delta \chi^{i}=0, \quad \delta u^{i}=i \varepsilon \chi^{i}
$$

for $u$ and $\chi$. Evidently, these are such that for any two fermionic parameters $\eta$ and $\varepsilon$,

$$
\delta_{\eta} \delta_{\varepsilon} \Phi=0
$$

for $\Phi$ equal to $u$ or $\chi$. Equation (2.8) corresponds to $Q^{2}=0$, and we wish to achieve this relation for all fields. For $\delta \rho$ we postulate

$$
\delta \rho_{\alpha}{ }^{i}=\varepsilon\left(H_{\alpha}^{i}+\frac{i}{2} \varepsilon_{\alpha \beta}\left(D_{k} J^{i}{ }_{j}\right) \chi^{k} \rho^{\beta j}\right)-i \varepsilon \Gamma_{j k}^{i} \chi^{j} \rho_{\alpha}{ }^{k} .
$$

This choice is not as arbitrary as it may at first appear. The term proportional to $D_{k} J^{i}{ }_{j}$ is needed for consistency with (2.4) and (2.7). The non-covariant looking term proportional to the affine connection is in fact needed for covariance under reparametrization of the $u^{i}$. So only the first term, $\varepsilon H_{\alpha}^{i}$ requires explanation. In fact, this may be regarded as the definition of $H^{i x}$; any terms that might be added to the right-hand side of (2.9) could be absorbed in redefinition of $H$.

Now we must ask what $\delta H$ should be. This is uniquely determined by asking that $\delta_{\eta} \delta_{\varepsilon} \rho$ should vanish. One finds that one must postulate the unpromisinglooking formula,

$$
\begin{aligned}
\delta H^{\alpha i}= & -\frac{\varepsilon}{4} \chi^{k} \chi^{l}\left(R_{k l t}{ }^{i}+R_{k l i^{\prime} t^{\prime}} J^{i^{\prime} i} J_{t}^{t^{\prime}}\right) \rho^{\alpha t}+\frac{i \varepsilon}{2} \varepsilon_{\beta}^{\alpha}\left(D_{k} J^{i}{ }_{j}\right) \chi^{k} H^{\beta j} \\
& -\frac{\varepsilon}{4}\left(\chi^{k} D_{k} J_{s}^{i}\right)\left(\chi^{l} D_{l} J_{t}^{s}\right) \rho^{\alpha t}-i \varepsilon \Gamma_{j k}^{i} \chi^{j} H^{\alpha k} .
\end{aligned}
$$

After a more or less lengthy calculation, one finds that

$$
\delta_{\eta} \delta_{\varepsilon} H=0
$$

as desired. This completes the construction of the fermionic multiplet. We will sometimes write formulas such as (2.9) or (2.10) in a different way, defining an operation $\{Q$,$\} on fields by \delta \Phi=-i \varepsilon\{Q, \Phi\}$, for all $\Phi$.

Because the algebra has been closed without the use of equations of motion, it is quite easy to find invariant actions. One simply takes

$$
\mathscr{L}=-i\{Q, V\}
$$

for any $V$. Because $Q^{2}=0$, the Lagrangian $\mathscr{L}$ is automatically invariant. We would like $\mathscr{L}$ to be conformally invariant and of $U=0$, so $V$ should be conformally invariant and of $U=-1$. A minimal choice is

$$
Z=\int d^{2} \sigma\left(\rho_{i}^{\alpha} \partial_{\alpha} u^{i}-\frac{1}{4} \rho_{i}^{\alpha} H_{\sigma}^{i}\right) \text {. }
$$


(We are here suppressing the world sheet metric, setting $h_{\alpha \beta}=\delta_{\alpha \beta}$. This can be done locally with the use of conformal invariance, and since the formulas are local this should cause no ambiguity.) By evaluating (2.12), we find the Lagrangian

$$
\begin{aligned}
\mathscr{L}=\int & d^{2} \sigma\left(-\frac{1}{4} H^{\alpha i} H_{\alpha i}+H_{i}^{\alpha} \partial_{\alpha} u^{i}-i \rho_{i}^{\alpha}\left(D_{\alpha} \chi^{i}+\frac{i}{2} \varepsilon_{\alpha \beta} \chi^{k} D_{k} J_{j}^{i} \partial^{\beta} u^{j}\right)\right. \\
& \left.-\frac{1}{8} \rho_{i}^{\alpha} \rho_{\alpha t} \chi^{k} \chi^{l} R_{k l}{ }^{i t}-\frac{1}{16} \rho_{i}^{\alpha} \rho_{\alpha t}\left(\chi^{k} D_{k} J_{s}^{i}\right)\left(\chi^{l} D_{l} J^{t s}\right)\right) .
\end{aligned}
$$

The derivative of $H$ does not appear in (2.14), so $H$ is an "auxiliary field," important in closing the algebra but not propagating. Bearing in mind (2.5), one finds the Euler-Lagrange equations to be

$$
H_{x}^{i}=\partial_{\alpha} u^{i}+\varepsilon_{\alpha \beta} J_{j}^{i} \partial^{\beta} u^{j} .
$$

Eliminating $H$ with the use of this equation, one finds a Lagrangian for the propagating fields only, namely

$$
\begin{aligned}
\mathscr{L}= & \int d^{2} \sigma\left[\frac{1}{2} g_{i j} \partial_{\alpha} u^{i} \partial^{\alpha} u^{j}+\frac{1}{2} \varepsilon^{\alpha \beta} J_{i j} \partial_{\alpha} u^{i} \partial_{\beta} u^{j}-i \rho^{\alpha} i\left(D_{\alpha} \chi^{i}+\frac{1}{2} \varepsilon_{\alpha \beta}\left(D_{k} J^{i} j\right) \chi^{k} \partial^{\beta} u^{j}\right)\right. \\
& \left.-\frac{1}{8} \chi^{k} \chi^{l} \rho^{\alpha}{ }_{i} \rho_{\alpha t} R_{k l}{ }^{i t}-\frac{1}{16} \chi^{k} \chi^{l} \rho^{\alpha i} \rho_{\alpha l}\left(D_{k} J_{j i}\right)\left(D_{l} J^{j t}\right)\right] .
\end{aligned}
$$

For future reference, let us here note the formula for the supercurrent that generates the fermionic symmetry. There is a standard recipe for finding this. One considers a fermionic transformation with a general $\sigma^{\alpha}$-dependent parameter $\varepsilon$. Writing the variation of the Lagrangian as $\delta \mathscr{L}=i \int d^{2} \sigma \partial_{\alpha} \varepsilon \cdot J^{\alpha}$, one finds the conserved current

$$
J^{\alpha}=g_{i j} H^{\alpha i} \chi^{j}+\frac{1}{2} J^{l s} \rho_{s}^{\alpha} D_{k} J_{i j} \chi^{k} \chi^{j} .
$$

2.1 The Kahler Case. The above formulas simplify and become more transparent if $M$ is a Kahler manifold $K$, so that $D_{k} J^{i}{ }_{j}=0$. Let us denote vector fields of type $(1,0)$ or $(0,1)$ on the Kahler manifold $K$ as $v^{I}$ and $w^{\bar{I}}$, respectively. The nonzero components of the Kahler metric $g$ are then $g_{I \bar{J}}=g_{\bar{J} I}$. Similarly, a one form $\omega_{\alpha}$ on the Riemann surface $\Sigma$ has components of type $(1,0)$ and $(0,1)$, which we may denote as $\omega_{+}$and $\omega_{-}$. Equation (2.4) means that the non-zero components of $\rho_{+}{ }^{i}$ and $\rho_{-}{ }^{j}$ are $\rho_{+}{ }^{I}$ and $\rho_{-}{ }^{\bar{J}}$, respectively. Equation (2.16) reduces to

$$
\begin{aligned}
\mathscr{L}= & 2 \int d^{2} \sigma\left[g_{I \bar{J}} \partial_{+} u^{I} \partial_{-} u^{\bar{J}}-\frac{i}{2} \rho_{+}{ }^{I} D_{-} \chi^{\bar{J}} g_{I \bar{J}}\right. \\
& \left.-\frac{i}{2} \rho_{-}{ }^{\widetilde{J}} D_{+} \chi^{I} g_{I \bar{J}}-\frac{1}{4} \chi^{I} \chi^{\bar{I}} \rho_{+}{ }^{J} \rho_{-}{ }^{\bar{J}} R_{I \bar{I} J \bar{J}}\right] .
\end{aligned}
$$

This possesses an $N=2$ fermionic symmetry

$$
\begin{aligned}
\delta \chi^{I} & =\delta \chi^{\bar{I}}=0, \quad \delta u^{I}=i \varepsilon \chi^{I}, \quad \delta u^{\bar{I}}=i \tilde{\varepsilon} \chi^{\bar{I}}, \\
\delta \rho_{+}{ }^{I} & =2 \tilde{\varepsilon} \partial_{+} u^{I}-i \varepsilon g^{I \bar{S}} \partial_{S} g_{K \bar{S}} \chi^{S} \rho_{+}{ }^{K}, \\
\delta \rho_{-}{ }^{\bar{I}} & =2 \varepsilon \partial_{-} u^{\bar{I}}-i \tilde{\varepsilon} g^{S \bar{I}} \partial_{\bar{S}} g_{S \bar{K}} \chi^{\bar{S}} \rho_{-}{ }^{\bar{K}} .
\end{aligned}
$$

Here $\varepsilon$ and $\tilde{\varepsilon}$ are independent anticommuting constants, which correspond to two fermionic charges $Q_{L}$ and $Q_{R}$ which obey $0=Q_{L}^{2}=Q_{R}^{2}=\left\{Q_{L}, Q_{R}\right\}$. For $\varepsilon=\tilde{\varepsilon}$, 
(2.19) reduces to the single fermionic symmetry of the more general model (2.10). $Q_{L}$ and $Q_{R}$ generate independent BRST-like symmetries for left and right moving modes. Similarly, the global symmetry $U$ of (2.16) splits into $U_{L} \times U_{R}$, with the charges of $\chi^{I}, \chi^{\bar{I}}, \rho_{+}{ }^{I}$, and $\rho_{-}{ }^{\bar{I}}$ being $(1,0),(0,1),(-1,0)$, and $(0,-1)$, respectively.

Unlike (2.16), (2.18) can be understood in a simple way as a twisted version of a standard $N=2$ supersymmetric nonlinear sigma model. In two dimensions the Lorentz group has a single generator which we will call $T$. The standard $N=2$ model (for maps into a Kahler manifold) has left and right moving ( $V-A$ and $V+A$ ) chiral symmetries which we may call $U_{L}$ and $U_{R}$. There are four supercharges (two left moving and two right moving); they transform under $T \times U_{L} \times U_{R}$ as $(-1 / 2,1,0) \oplus(-1 / 2,-1,0) \oplus(1 / 2,0,1) \oplus(1 / 2,0,-1)$. Clearly, if we define a new Lorentz generator $T^{\prime}=T+\frac{1}{2} U_{L}-\frac{1}{2} U_{R}$, then there are two $T^{\prime}$ singlet supercharges $Q_{L}$ and $Q_{R}$, transforming under $U_{L} \times U_{R}$ as $(1,0) \oplus(0,1)$. They obey $Q_{L}^{2}=Q_{R}^{2}=\left\{Q_{L}, Q_{R}\right\}=0$. With $T^{\prime}$ regarded as the Lorentz generators, the fermions have spin 0 and \pm 1 instead of $\frac{1}{2}$ and $-\frac{1}{2}$. The resulting model can be defined on an arbitrary Riemann surface preserving the conservation of $Q_{L}$ and $Q_{R}$. This gives precisely (2.18).

It is also possible, if $M$ is a Kahler manifold, to consistently set $\rho_{-}{ }^{J}=\chi^{\bar{J}}=0$, leaving a Lagrangian

$$
\mathscr{L}=2 \int d^{2} \sigma\left[g_{I \bar{J}} \partial_{+} u^{I} \partial_{-} u^{\bar{I}}-\frac{i}{2} \rho_{-}{ }^{\bar{J}} D_{+} \chi^{I} g_{I \bar{J}}\right]
$$

with a single right moving fermionic symmetry

$$
\begin{aligned}
\delta \chi^{I} & =0=\delta u^{\bar{I}}, \quad \delta u^{I}=i \varepsilon \chi^{I}, \\
\delta \rho_{-}{ }^{\bar{I}} & =2 \varepsilon \partial_{-} u^{\bar{I}} \\
\delta \rho_{+}{ }^{\bar{I}} & =-i \varepsilon g^{l \bar{S}} \cdot \partial_{S} g_{K \bar{S}} \chi^{S} \rho_{+}{ }^{K}
\end{aligned}
$$

This is a twisted version of a conventional $(0,2)$ model. Though the Kahler case is thus relatively transparent the general Lagrangian (2.16) for almost complex manifolds apparently cannot be obtained by twisting of any more standard construction.

2.2 Incorporation of World-Sheet Supersymmetry. In this section, we will generalize the above constructions to give models with $1+1$-dimensional world-sheet supersymmetry as well as BRST invariance. The main motivation has to do with the conjecture that the sigma models we have described, in which the graviton vertex operator turns out to be a BRST commutator, are related to an unbroken phase of string theory. If so, the above models are related to an unbroken phase of bosonic strings; sigma models related to an unbroken phase of Type II or heterotic superstrings should have $N=1$ or $N=1 / 2$ world-sheet supersymmetry, respectively, as well as BRST symmetry, and we would like to construct these sigma models.

First we recall the basic definitions of $N=1$ superspace in $1+1$ dimensions. In addition to the bosonic coordinates $\sigma^{\alpha}, \alpha=1,2$, there are fermionic coordinates 
$\theta^{A}, A=1,2$ which transform as spinors of the Lorentz group. ${ }^{5}$ The supersymmetry generators are

$$
Q_{A}=\frac{\hat{\partial}}{\partial \theta^{A}}+i\left(\rho^{\alpha} \theta\right)_{A} \frac{\partial}{\partial \sigma^{\alpha}}
$$

The superspace covariant derivative is

$$
D_{A}=\frac{\partial}{\partial \theta^{A}}-i\left(\rho^{\alpha} \theta\right)_{A} \frac{\partial}{\partial \sigma^{\alpha}} .
$$

The relation between them is $\left\{Q_{A}, D_{B}\right\}=0$. A superfield is simply a function $\Phi\left(\sigma^{\alpha}, \theta^{A}\right)$, with the supersymmetry transformation law $\delta \Phi=\varepsilon^{A} Q_{A} \Phi$. Any expression $\int d^{2} \sigma d^{2} \theta P\left(\Phi, D_{A} \phi, D_{A} D_{B} \phi, \ldots\right)$ is supersymmetric, with $P$ being an arbitrary functional of $\Phi$ and its covariant derivatives. A typical (in some sense minimal) choice is

$$
\mathscr{L}=\int d^{2} \sigma d^{2} \theta \varepsilon^{A B} D_{A} \Phi D_{B} \Phi
$$

While supersymmetry may be realized in this way on any superfield or collection of superfields, we wish to find a particular collection of superfields on which one can realize the BRST-like fermionic symmetry in addition to supersymmetry.

The form in which we closed the algebra makes it easy to do this, essentially by re-interpreting the above fields as superfields and making some slight adjustments. Corresponding to the fields $u^{i}$ and $\chi^{i}$ of the previous construction, we introduce superfields $\hat{u}^{i}$ and $\hat{\chi}^{i}$ (commuting and anti-commuting, respectively; and of conformal spin zero), with the BRST transformation laws

$$
\delta \hat{\chi}^{i}=0, \quad \delta \hat{u}^{i}=i \varepsilon \hat{\chi}^{i} .
$$

As for $\rho^{\alpha i}$ and $H^{\alpha i}$, we replace them with "spinor" superfields $\hat{\rho}^{A i}$ and $\hat{H}^{A i}$ of conformal spin $1 / 2$, and obeying the kinematical constraints

$$
\hat{\rho}^{A i}=\varepsilon^{A}{ }_{B} J_{j}^{i} \hat{\rho}^{B j}, \quad \hat{H}^{A i}=\varepsilon^{A}{ }_{B} J^{i}{ }_{j} \hat{H}^{B j} .
$$

The BRST transformation laws are the obvious generalizations of our previous ones,

$$
\begin{aligned}
\delta \hat{\rho}^{A i}= & \varepsilon \hat{H}^{A i}+\frac{i \varepsilon}{2} \varepsilon^{A}{ }_{B} \hat{\chi}^{k} D_{k} J_{j}^{i} \hat{\rho}^{B j}-i \varepsilon \Gamma_{j k}^{i} \hat{\chi}^{j} \hat{\rho}^{A k} \\
\delta \hat{H}^{A i}= & -\frac{\varepsilon}{4} \hat{\chi}^{k} \hat{\chi}^{l}\left(R_{k l t}{ }^{i}+R_{k l i^{\prime} t^{\prime}} J^{i^{\prime} i} J^{t^{\prime} t}\right) \hat{\rho}^{A t}+\frac{i \varepsilon}{2} \varepsilon^{A}{ }_{B} \hat{\chi}^{k} D_{k} J^{i}{ }_{j} \hat{H}^{B j} \\
& -\frac{\varepsilon}{4}\left(\hat{\chi}^{k} D_{k} J^{i}{ }_{s}\right)\left(\hat{\chi}^{l} D_{l} J_{t}{ }^{s}\right) \hat{\rho}^{A t}-i \varepsilon \Gamma_{j k}^{i} \hat{\chi}^{j} \hat{H}^{A k}
\end{aligned}
$$

\footnotetext{
${ }^{5}$ This is a two dimensional real irreducible representation of $S O(2)$. It possesses both symmetric and antisymmetric bilinear invariants $\delta_{A B}$ and $\varepsilon_{A B}$. The former is used to "raise and lower" spinor indices. The complexification of this representation splits as a sum of one dimensional representations of "positive and negative chirality." The product of two spinor representations of $S O(2)$ contains a vector; this is expressed in the existence of the "gamma matrices" $\rho_{A B}^{\alpha}$
} 
This algebra closes by exactly the same computation which shows such closure in the case that the world sheet is purely bosonic. We then can find a supersymmetric and BRST invariant Lagrangian in the form

$$
\mathscr{L}=\left\{Q_{\text {BRST }}, \hat{V}\right\},
$$

where $\hat{V}$ is an arbitrary functional of the fields. A simple generalization of the choice that we adopted in the absence of world-sheet supersymmetry is

$$
\hat{V}=\int d^{2} \sigma d^{2} \theta\left(\hat{\rho}_{i}^{A} D_{A} \hat{u}^{i}-\frac{1}{4} \hat{\rho}_{i}^{A} \hat{H}_{A}^{i}\right) .
$$

The Lagrangian in (2.28) can be regarded as a supersymmetric generalization of (2.16), and if (2.16) has something to do with an unbroken phase of bosonic strings, then (2.28) may well have something to do with an unbroken phase of Type II superstrings. In a similar fashion, by introducing superfields that obey suitable chirality conditions, one can construct a generalization of (2.16) with $N=1 / 2$ world-sheet supersymmetry, possibly related to an unbroken phase of heterotic strings. The key point in introducing chiral superfields with BRST symmetry is that the BRST transformation laws (2.9), (2.10) still make sense and still close if one introduces chirality conditions $\hat{\rho}^{\alpha i}= \pm i \varepsilon_{\beta}^{\alpha} \hat{\rho}^{\beta i}, \hat{H}^{\alpha i}= \pm i \varepsilon_{\beta}^{\alpha} \hat{H}^{\beta i}$. Similar chirality conditions are possible in (2.26) and (2.27).

2.3 Local Fermionic Symmetry. Another interesting generalization of the forgoing involves the possibility of turning the global BRST symmetry that we have considered into a local symmetry with a $\sigma$-dependent infinitesimal parameter $\varepsilon$. (In carrying out this step, we will dispense with world-sheet supersymmetry and return to purely bosonic world-sheets.) At first sight, introducing local BRST symmetry might seem like a rather bizarre step. The motivation for this step is that if the BRST-like symmetries considered here (and in $[4,10]$ ) have anything to do with nature, they must be spontaneously broken so that local physics can emerge. Spontaneous breaking of BRST symmetry is at first sight a somewhat paradoxical notion. For the usual criterion of symmetry breaking is that a symmetry is broken if the vacuum is not invariant under the symmetry. In the case of BRST symmetry, the vacuum not being invariant under the symmetry would merely seem to mean that the vacuum is unphysical, in the BRST sense - not a very appealing conclusion. It seems likely that spontaneous BRST symmetry breaking would make more sense in the case of local BRST invariance. One would like to consider local BRST symmetry in space-time ( $3+1$ dimensions) as well as the string world sheet $(1+1$ dimensions), but as a first step we will here consider the $1+1$ dimensional case.

In constructing a Lagrangian with local BRST symmetry, the first point is that $\sigma$ derivatives nowhere appear in the transformation laws (2.7), (2.9), and (2.10). Consequently, those formulas still make sense and still give a closed algebra if $\varepsilon$ has an arbitrary $\sigma$ dependence. Any functional $\{Q, V\}$ will have local BRST symmetry if $V$ is constructed only from the fields $u, \chi, \rho$, and $H$, and not their derivatives. But if we wish to construct invariant functionals that involve not only the fields but also their derivatives, we need to introduce covariant derivatives. This may be done by introducing an anticommuting gauge field $\Lambda_{\alpha}$, of ghost 
number -1 , with the BRST transformation law

$$
\delta \Lambda_{\alpha}=-\partial_{\alpha} \varepsilon
$$

Then we define the covariant derivative of $u^{i}$ by

$$
D_{\alpha} u^{i}=\partial_{\alpha} u^{i}+i \Lambda_{\alpha} \chi^{i}
$$

This transforms homogeneously under $\sigma$ dependent BRST transformations (that is, under (2.30) together with (2.7)). The covariant derivative of $\chi$ needs no modification, since $\delta \chi=0$ in (2.7). Covariant derivatives of $\rho$ and $H$ receive modifications similar to that in (2.31) (so $D_{\alpha} \rho$ is replaced by $D_{\alpha} \rho-i \Lambda_{\alpha}\{Q, \rho\}$, etc.).

Now there is no problem in obtaining a generalization of our previous Lagrangians with local BRST symmetry. One simply replaces $\hat{o}_{x} u^{i}$ in (2.13) with $D_{\alpha} u^{i}$. After evaluating $\{Q, V\}$ and eliminating $H$, one arrives at a version of (2.16) with local fermionic symmetry in which, again, $\partial_{\alpha} u^{l}$ has been replaced by the covariant derivative.

To complete the picture, we should introduce a locally BRST invariant kinetic energy for the gauge field $\Lambda_{\alpha}$. One way to do this is certainly to introduce another anticommuting gauge field $\tilde{\Lambda}^{\alpha}$, of $U=+1$, invariant under the BRST symmetry (but with its own gauge invariance), and with the Lagrangian

$$
\mathscr{L}_{\text {gauge }}=-\frac{1}{4} \int d^{2} \sigma\left(\hat{\partial}_{\alpha} \Lambda_{\beta}-\partial_{\beta} \Lambda_{\alpha}\right)\left(\partial^{\alpha} \tilde{\Lambda}^{\beta}-\partial^{\beta} \tilde{\Lambda}^{\alpha}\right) .
$$

This is not suitable for our general program, however, since the energy-momentum tensor is not a BRST commutator, so that (2.32) cannot be interpreted in terms of an unbroken phase of quantum gravity. Construction of a more suitable version and investigation of its properties is something that we will leave for the future.

2.4 Families of Almost Complex Manifolds. Our considerations up to this point have still a further generalization, which is likely to be important in geometrical applications, though it will not really be exploited in this paper. This generalization again depends on the fact that $\sigma$ derivatives of the fields do not appear in the fermionic transformation laws (2.7), (2.9), and (2.10). As a result, the algebra will still close if with no other change in any of the formulas, we permit $g_{i j}\left(u^{k} ; \sigma^{\alpha}\right)$ and $J^{i}{ }_{j}\left(u^{k} ; \sigma^{\alpha}\right)$ to depend on the coordinates $\sigma^{\alpha}$ of $\Sigma$ as well as the coordinates $u^{k}$ of $M$. Our Lagrangian (2.16) is still invariant in this more general situation.

To make this sound a little less bizarre, and describe its geometric setting, let us introduce the product space $X=M \times \Sigma . X$ is an almost complex manifold, since $M$ is almost complex and $\Sigma$ is a Riemann surface. $X$ can be viewed as a fiber bundle over $\Sigma$,

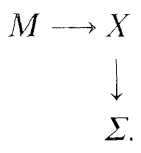

The space of maps $\Sigma \rightarrow M$ can be viewed as the space of sections of the bundle (2.33). Of course, $X=M \times \Sigma$ is a trivial bundle. Nevertheless, let us pick on $X$ a metric and almost complex structure which are not simply products of structures coming from $M$ and $\Sigma$. (We require the almost complex structure on $X$ to be 
compatible with the fibration in (2.33) in the sense that the tangent bundle to the fibers, viewed as a subbundle of the total tangent bundle of $X$, is invariant under the action of the endomorphism that defines the complex structure of $X$.) Such a choice of metric and almost complex structure on $X$ will restrict on each fiber to a metric and complex structure $g_{i j}\left(u^{k} ; \sigma^{\alpha}\right)$ and $J_{j}^{i}\left(u^{k} ; \sigma^{\alpha}\right)$ on $M$ with, in general, a non-trivial dependence on the coordinates $\sigma^{\alpha}$ of $\Sigma$.

At this point, one might feel that it is rather unnatural to consider only product bundles while permitting a non-product metric and a non-product almost complex structure. Clearly, the situation just described is begging to be generalized to a setting in which we replace the maps $\Sigma \rightarrow M$ by sections of an arbitrary $M$ bundle over $\Sigma$. To obtain the correct general setting for topological sigma models, we should consider an arbitrary family of almost complex manifolds, fibered over a Riemann surface $\Sigma$. By a family of almost complex manifolds, we mean a manifold $X$ fibered over $\Sigma$, with an almost complex structure on $X$ that reduces for each fiber to an almost complex structure on the fiber. We then regard the $u^{k}\left(\sigma^{\alpha}\right)$ as a description not of a map $\Sigma \rightarrow M$ but of a section of the bundle $X . X$ is then endowed with a metric of type $(1,1)$--that is, a metric obeying (2.4). With slight modification, (2.16) is still well-defined in this situation, and still possesses its fermionic symmetry.

Modification is required because, with the $u^{k}\left(\sigma^{\alpha}\right)$ being not functions but sections of a bundle, the derivatives $\partial_{\alpha} u^{k}$ must be replaced by suitable covariant derivatives. What will be the "gauge group" in the definition of these covariant derivatives? In general there is no reason for this group to be any smaller than the full-fledged group Diff $M$ of all diffeomorphisms of $M$. Let $\operatorname{diff} M$ be the Lie algebra of this group, and let $V_{a}, a \in T$, be a basis of this Lie algebra. Of course, the indexing set $T$ is infinite, as $\operatorname{diff} M$ is an infinite dimensional Lie algebra. Concretely, each $V_{a}$ corresponds to a vector field $V_{a}^{i}$ on $M$. We now wish to introduce a connection on $X$ with Diff $M$ for structure group. Concretely, this means introducing on $\Sigma$ a gauge field $A_{\alpha}{ }^{a}\left(\sigma^{\beta}\right)$ which, locally, can be regarded as a diff $M$ valued one form, with the usual gauge transformation law

$$
\delta A_{\alpha}{ }^{a}=-\partial_{\alpha} \varepsilon^{a}+f^{a}{ }_{b c} \varepsilon^{b} A_{\alpha}^{c}
$$

with $f_{b c}^{a}$ the structure constants of $\operatorname{diff} M$ (so $\left[V_{b}, V_{c}\right]=f_{b c}^{a} V_{a}$ ) and $\varepsilon^{a}$ being an arbitrary $\operatorname{diff} M$ valued zero form. Now we can define the covariant derivative of the $u^{k}$

$$
D_{\alpha} u^{k}=\partial_{\alpha} u^{k}+\Sigma_{a \in T} A_{\alpha}{ }^{a} V_{a}{ }^{k},
$$

and a corrected covariant derivative of the $\chi^{k}$,

$$
D_{\alpha} \chi^{k}=\partial_{\alpha} \chi^{k}+\partial_{\alpha} u^{i} \Gamma_{i l}^{k} \chi^{l}+\Sigma_{a \in T} A_{\alpha}^{a} \partial_{j} V_{a}^{k} \chi^{j} .
$$

If the derivatives of $u^{k}$ and $\chi^{k}$ are everywhere replaced by (2.35) and (2.36), then (2.16) makes sense in the more general context just described, and is still invariant under the fermionic symmetry (2.9). Thus, we have obtained a "supersymmetric" theory describing the sections of an arbitrary family $X \rightarrow \Sigma$ of almost complex manifolds. The "instantons" in this situation are the global holomorphic sections of the family. 
In case $X$ and $M$ are Kahler, the discussion simplifies. Perhaps it is appropriate to discuss this case explicitly. The nonlinear sigma model is usually (without the generalization we have just discussed) a theory of maps $\Sigma \rightarrow M$. As such, it depends on the intrinsic geometry of $M$, not on the choice of a particular set of coordinates $u^{k}$. The intrinsic nature of the nonlinear sigma model shows up in the fact that the usual Lagrangian

$$
\mathscr{L}=\frac{1}{2} \int d^{2} \sigma g_{k l} \partial_{\alpha} u^{k} \partial^{\alpha} u^{l}
$$

preserves its form under reparametrizations at the $u^{k}$-under, say, $u^{k} \rightarrow \tilde{u}^{l}\left(u^{k}\right)$, accompanied by the usual redefinition of $g_{k l}$. Of course, if one is considering sigma models with an additional structure, say a Kahler structure permitting an $n=2$ supersymmetry, one should consider only reparametrizations that preserve this additional structure. In any case, (2.37) would not preserve its form under a $\sigma^{\alpha}$ dependent reparametrization $u^{k} \rightarrow \tilde{u}^{l}\left(u^{k} ; \sigma^{\alpha}\right)$. Of course, we could generalize (2.37), by including a $\operatorname{diff} M$ connection as above, to a model describing sections of a not necessarily trivial bundle $X \rightarrow \Sigma$. Usually such a generalization would not be compatible with fermionic symmetries. The surprise about $(2.16)$ is that the fermionic symmetry holds in the more general situation.

This is particularly transparent in the Kahler situation (2.18). Let us write $z$ and $\bar{z}$ for $\sigma^{+}$and $\sigma^{-}$. Note that in (2.18) we see $\partial_{z} u^{I}$ and $\partial_{z} u^{I}$, but no $\partial_{z} u^{I}$ or $\partial_{z} u^{\bar{I}}$. Therefore, (2.18) preserves its form under holomorphic reparametrizations of the $u^{I}$ that depend holomorphically on $z$, say

it being essential that

$$
u^{I} \rightarrow \tilde{u}^{I}\left(u^{J} ; z\right)
$$

$$
\frac{\partial \tilde{u}^{I}}{\partial u^{I}}=\frac{\partial \tilde{u}^{I}}{\partial \bar{z}}=0 .
$$

The fact that (2.18) preserves its form under (2.38) means that (2.38) has a generalization to a situation in which one is studying the sections of a family

$$
\begin{array}{r}
M \longrightarrow X \\
\downarrow \\
\Sigma
\end{array}
$$

of Kahler manifolds fibered over $\Sigma$, rather than maps $\Sigma \rightarrow M$. This also holds in the more general case of sections of a family of almost complex manifolds, corresponding to the more general Lagrangian (2.16).

In this section, we have considered a model that involves world sheet gravity (an arbitrary metric on $\Sigma$ ) and world sheet gauge fields (the $\operatorname{diff} M$ connection). But these play a passive role, being arbitrarily prescribed rather than being independent degrees of freedom. In later sections, we will generalize to the case of a sigma model coupled to dynamical gravity and dynamical gauge fields, still possessing the fermionic symmetry.

2.5 Canonical Formulation and Floer Groups. Finally, we will, very briefly, discuss the canonical quantization of (2.16). Such canonical quantization will give rise to 
a quantum Hilbert space $\mathscr{H}$, with an operator $Q$ obeying $Q^{2}=0$. It is to be expected that in a suitable setting, the $U$-graded cohomology groups of $Q$ will turn out to be the Floer groups. Recall, after all, that the original motivation for the construction of (2.16) was to find a relativistic generalization of the de Rham model of $1+1$ dimensional Floer theory, as described in [3].

A full discussion of the quantization of (2.16) would raise many issues. Here, we will simply make a few remarks. First of all, in (2.16) there appears a certain term

$$
\Delta \mathscr{L}=\frac{1}{2} \int d^{2} \sigma J_{i j} \varepsilon^{\alpha \beta} \partial_{\alpha} u^{i} \partial_{\beta} u^{j} .
$$

It is clear from the CPT theorem that this term, because it is odd under reversal of orientation of $\Sigma$ but appears with a real coefficient, will cause certain difficulties in quantization. The CPT theorem says that the (Euclidean) Lagrangian of a unitary, relativistic quantum field theory must be invariant under complex conjugation combined with reversal of orientation. Physically, in fact, (2.41) corresponds to a world-sheet $\theta$ term with an imaginary value of $\theta$, and this certainly violates unitarity.

In Atiyah's treatment of Floer theory [3], there does not seem to be any sign of an imaginary theta term, so we are led to ask if (2.41) can be omitted from (2.16) without spoiling the fermionic symmetry. There is one situation is which this is possible. If the two form $J=\frac{1}{2} J_{i j} d u^{i} \wedge d u^{j}$ is closed, $d J=0$, then (2.41) is a topological invariant and can be dropped without spoiling the fermionic symmetry. The case $d J=0$ (by definition) the case in which $M$ is symplectic and not just almost complex. This is precisely the situation most investigated by Floer, whose $1+1$ dimensional results are mainly concerned with diffeomorphisms of symplectic manifolds. It is conceivable that Floer theory has a generalization to the almost complex case. Whether or not such a generalization exists, it seems likely that the theorems that Floer has actually obtained correspond most closely to quantization of (2.16) with $M$ symplectic and (2.41) omitted.

One may note that even if (2.41) is omitted, (2.16) still leads to a Lorentz invariant inner product on the quantum Hilbert space $\mathscr{H}$ which is not unitary. The non-unitarity appears because of the indefiniteness of the fermion kinetic energy. In [4], a similar problem arose, and it was noted that in the Hamiltonian formalism there is a time reversal symmetry that enables one to define a positive definite but not Lorentz invariant inner product. Equation (2.16) does not have such a symmetry. It is possible that by adding non-minimal BRST invariant terms (of the form $\left\{Q, V^{\prime}\right\}$ for suitable $\left.V^{\prime}\right\}$ one can find a version that admits a time reversal symmetry, but this question will not be addressed here.

Now, the situation that Floer actually considers is that of a symplectic manifold $M$ together with a diffeomorphism $\phi: M \rightarrow M$ which preserves the symplectic structure. From our point of view, the most natural way to study this situation is to regard $\phi$ as the gluing data in constructing a certain $M$ bundle over $S^{1}$, that is, a manifold $Y$ fibered over $S^{1}$, the fibers being copies of $M$. Then, instead of maps $S^{1} \rightarrow M$, we consider sections of the bundle $Y$. More exactly, in the Hamiltonian approach one considers an $M$-bundle $Y$ over $S^{1}$. In the path integral approach, one must restore the time direction, and thus consider the product $Y \times R ; R$ is the "time-line," and $X=Y \times R$ is regarded as an $M$ bundle over $S^{1} \times R$. In view 
of our discussion in the last subsection, (2.16) gives a BRST invariant theory governing the sections of the bundle $Y$ over $S^{1} \times R .{ }^{6}$ It is the quantization of (2.16), in this sense, that should be expected to give the groups that Floer associates with a symplectic diffeomorphism $\phi: M \rightarrow M$.

In fact, in the Hamiltonian picture, the quantum ground states correspond to minima of the energy. The purely bosonic part of the energy is

$$
H_{B}=\frac{1}{2} \int_{0}^{2 \pi} d \sigma\left(g_{i j} \frac{d u^{i}}{d \tau} \frac{d u^{j}}{d \tau}+g_{i j} \frac{d u^{i}}{d \sigma} \frac{d u^{j}}{d \sigma}\right) .
$$

(Here $\tau$ is "time," and $S^{1}$ is being parametrized with an angular variable $\sigma$ running from 0 to $2 \pi$.) If present, (2.41) would contribute an extra term to the energy, but this has been omitted. (At this point it is crucial that $M$ is symplectic and (2.41) is being deleted. The additional term in the energy makes a contribution which is not positive and would modify the determination of the Floer groups.) To minimize (2.42) in the classical approximation, one takes $u^{i}$ to be constant. If, however, one is considering not maps $S^{1} \rightarrow M$ but sections of a bundle constructed with the diffeomorphism $\phi$ as the transition function, then the boundary conditions on $u^{i}$ are not $u^{i}(2 \pi)=u^{i}(0)$, but

$$
u^{i}(2 \pi)=\phi^{*}\left(u^{i}\right)(0)
$$

In other words, the $u^{i}$ are periodic up to the diffeomorphism $\phi$. Equation (2.43) means that $u^{i}$ can be constant only if the constant value corresponds to a fixed point of $\phi$, and this is why the quantum ground states in the situation under discussion can given information about those fixed points. Presumably, in this way one can recover Floer's results about symplectic diffeomorphisms.

It seems appropriate to conclude by stating explicitly the following generalization of these considerations. This involves a situation that is known to have intimate analogies with number theory. Consider a Kahler manifold $X$ with a surjective holomorphic map $\pi: X \rightarrow \Sigma, \Sigma$ being a compact Riemann surface. It will always be true that over the complement of finitely many points "of bad reduction," $\pi$ is a fibration. Suppose that $\pi$ is everywhere a fibration. Then (2.16) gives a BRST invariant quantum field theory describing the $C^{\infty}$ sections of the bundle $X \rightarrow \Sigma$. The global holomorphic sections will play a special role, as we will see in the next section. In fact, we will introduce Donaldson-like invariants that can be computed in terms of the global holomorphic sections of this bundle. More generally, there will be finitely many points $P_{1} \cdots P_{k}$ at which the fibration $\pi$ is ill behaved. (And we may wish to permit the total space $X$ to have singularities lying above those points.) Around each of the $P_{j}$, draw a little circle $S_{j}$. The fibration $\pi: X \rightarrow \Sigma$ can be restricted to $S_{j}$ to give a one parameter family of Kahler manifolds fibered over $S_{j}$. By quantizing a sigma model governing the sections of this bundle one obtains

\footnotetext{
6 When one considers not maps $\Sigma \rightarrow M$, but sections of an $M$ bundle over $\Sigma$, the ability to drop (2.41) depends on finding a closed two form $\omega$ on the total space of the bundle whose restriction to the fibers induces the symplectic structures of the fibers. In the case at hand, this will exist if the diffeomorphism $\phi$ of $M$ is symplectic
} 
certain Floer groups, just as in our discussion in the last subsection (where we associated Floer groups with any one parameter family of symplectic manifolds fibered over a circle). We should think of these as the local Floer groups associated with the singularity at $P_{j}$. Because of the singularities at the $P_{j}$, to make sense of the quantum field theory associated with $\pi: X \rightarrow \Sigma$ will require suitable boundary conditions on the $S_{j}$, corresponding to the choice of a state in the local Floer groups. Thus, the Donaldson-like invariants which we will associate in the next section with global holomorphic sections of the bundle $X$ will - in this situationtake values in the local Floer groups associated with what number theorists would call the primes of bad reduction.

\section{Observables}

In this section, we will discuss the observables which can be defined in the model described in Sect. 2. We will restrict ourselves to the case in which one is studying maps $\Sigma \rightarrow M$ for some $M$ (rather than sections of a bundle). We will restrict ourselves to observables in the BRST sense. This means that we consider only operators $\mathcal{O}$ which are such that $\{Q, \mathcal{O}\}=0$. The corresponding correlation functions

$$
\left\langle\mathcal{O}_{1} \mathcal{O}_{2} \cdots \mathbb{O}_{n}\right\rangle
$$

are zero if any of the $\mathcal{O}_{i}$ are BRST commutators, that is, if any of them can be written as $\mathscr{C}_{i}=\{Q, \lambda\}$ for some $\lambda$. (The argument that (3.1) is zero if one of the $\mathcal{O}_{i}$ is a BRST commutator can be found in the string theory literature $[14,15]$ and has been reviewed in [4].) Thus, discussing the observables in the BRST sense requires us to consider BRST cohomology classes of operators-operators $\mathcal{O}$ obeying $\{Q, \mathcal{O}\}=0$ modulo operators of the form $\mathcal{O}=\{Q, \hat{\lambda}\}$.

Exactly as in [4], the only observables that can be defined in this sense are global topological observables. The reason for this is that the BRST-like symmetry is linearly realized; every field has a superpartner; the fermionic symmetry acts in a non-degenerate fashion even in a linearized approximation.

Since the Lagrangian $\mathscr{L}(2.16)$ possesses a fermionic symmetry for any choice of metric $h_{\alpha \beta}$ on $\Sigma$ and for any choice of metric $g_{i j}$ and complex structure $J^{i}{ }_{j}$ on $M$, the variation of $\mathscr{L}$ with respect to the metric or complex structure of $\Sigma$ or $M$ is BRST invariant. The characteristic features of "topological sigma models" spring from the fact that these particularly important BRST invariant operators are actually BRST commutators, that is, they can be written as $\{Q, X\}$ for some $X$. For instance, the two dimensional energy-momentum tensor is defined in terms of the change in $\mathscr{L}$ under an infinitesimal change in the two dimensional metric $h_{\alpha \beta}$ :

$$
\delta \mathscr{L}=\frac{1}{2} \int_{\Sigma} \sqrt{h} \delta h^{\alpha \beta} T_{\alpha \beta} .
$$

One finds that $T_{\alpha \beta}$ is a BRST commutator; in fact, $T_{\alpha \beta}=-i\left\{Q, \Lambda_{\alpha \beta}\right\}$ with

$$
\Lambda_{\alpha \beta}=\frac{1}{2}\left(g_{i j} \rho_{\alpha}^{i} \partial_{\beta} u^{j}+g_{i j} \rho_{\beta}^{i} \partial_{\alpha} u^{j}-h_{\alpha \beta} g_{i j} \rho^{\sigma i} \partial_{\sigma} u^{j}\right) .
$$


Actually, it is obvious without any computation at all that $T_{\alpha \beta}$ must be a BRST commutator. For as $\mathscr{L}$ itself is a BRST commutator (having been introduced that way in (2.12)), its variation under a change in the metric on $\Sigma$ is certainly a BRST commutator. For essentially the same reason, the change in $\mathscr{L}$ under a change in the metric and complex structure of $M$ is a BRST commutator. In fact, under a change in $g_{i j}$ and $J_{j}^{i}$, the change in $\mathscr{L}$ is

$$
\delta \mathscr{L}=\left\{Q, \int d^{2} \sigma\left(\rho^{\alpha i} \partial_{\alpha} u^{j} \cdot\left(\delta g_{i j}+\frac{1}{2}\left(\delta J^{t}{ }_{l} J_{j t}-\delta J^{t}{ }_{j} J_{l t}\right)\right)\right)\right.
$$

The change in the Lagrangian under a change in the metric of $M$ is known in string theory as the graviton vertex operator. The fact that the graviton vertex operator in the situation at hand is a BRST commutator is precisely the reason for the conjecture that these "topological sigma models" may give a realization in string theory of a phase with unbroken general covariance. It means that the metric fluctuation is not observable in the BRST sense, and therefore that even the value of the metric is not observable. (This has nothing to do with quantum fluctuations; it is true in the phase at hand even in the classical limit.) This is exactly what one would expect if one is studying an underlying generally covariant theory, in a phase in which general covariance is a symmetry of the vacuum although violated by the BRST gauge fixing.

3.1 Global Observables. The BRST invariant operators that are not BRST commutators are--as in [4]-- of a global topological nature. Recall that in [4], the key was the existence of a BRST invariant field $\phi$ of conformal spin zero. Looking back to the fermionic transformation law (2.7), we see that the closest analogue of that in the present discussion is the existence of a field $\chi$ of conformal spin zero with $\delta \chi^{i}=0$. However, since $\chi^{i}$ is not a function but a section of the bundle $\phi^{*}(T)$, it does not make sense to consider operators that "depend only on $\chi^{i}$ and not on the boson fields $u^{i}$." Such a concept would not be invariant under reparametrizations of the $u^{i}$. The natural notion is to consider a differential form, say an $n$-form $A=A_{i_{1} \cdots i_{n}} d u^{i_{1}} \cdots d u^{i_{n}}$, on $M$. For every such $n$-form $A$, we consider the operator

$$
\mathcal{O}_{A}^{(0)}=A_{i_{1} i_{2} \ldots i_{n}} \chi^{i_{1}} \chi^{i_{2}} \cdots \chi^{i_{n}}
$$

By use of (2.9), we find

$$
\delta \mathcal{O}_{A}^{(0)}=i \varepsilon \partial_{i_{0}} A_{i_{1} i_{2} \ldots i_{n}} \chi^{i_{0}} \chi^{i_{1}} \cdots \chi^{i_{n}}
$$

which (recalling the definition $\delta \mathcal{O}=-i \varepsilon\{Q, \mathcal{O}\}$ ) is equivalent to the statement that

$$
\left\{Q, \mathcal{O}_{A}^{(0)}\right\}=-\mathcal{O}_{\mathrm{d} A}^{(0)},
$$

where $d A=\partial_{i_{0}} A_{i_{1} \cdots i_{n}} d u^{i_{0}} d u^{i_{1}} \cdots d u^{i_{n}}$ is the exterior derivative of $A$. From (3.7), it is clear that BRST invariant operators $\mathcal{O}_{A}^{(0)}$ correspond exactly to closed forms $A$. Moreover, it is clear that $\mathcal{O}_{A}$ can be written as a BRST commutator, $\mathcal{O}_{A}=-\left\{Q, \mathcal{O}_{B}\right\}$, if and only if $A=d B$ is trivial in de Rham cohomology. Thus, the BRST cohomology classes of operators obtainable in this way correspond exactly to the de Rham 
cohomology classes of $M{ }^{7}$ Clearly, the degree of $\mathcal{O}_{A}$ under the $U$ grading is the same as the degree of $A$ as a differential form.

Now, recall the basic framework of correlation functions in quantum field theory. We pick a homotopy class of maps $\Sigma \rightarrow M$, and introduce the Feynman path integral

$$
\int \mathscr{D} X e^{-\mathscr{L}}
$$

with $\mathscr{D} X$ an abbreviation for an integral over all $u, \rho, \chi$ (in the chosen homotopy class), and $\mathscr{L}$ the Lagrangian of Eq. (2.16). For any functional $W$ of $u, \rho, \chi$, we define the unnormalized expectation value

$$
\langle W\rangle=\int \mathscr{D} X e^{-\mathscr{L}} W
$$

Its most important property is that

$$
(\{Q, U\})=0
$$

for any $U$.

We can now formulate some interesting examples. If $A_{1}, A_{2}, \ldots A_{k}$ are closed forms on $M$ of degrees $n_{1}, \ldots, n_{k}$, pick distinct points $P_{1}, \ldots, P_{k} \in \Sigma$, and consider

$$
Z\left(A_{1}, \ldots, A_{k}\right)=\left\langle\mathcal{O}_{A_{1}}^{(0)}\left(P_{1}\right) \mathcal{O}_{A_{2}}^{(0)}\left(P_{2}\right) \cdots \mathcal{O}_{A_{k}}^{(0)}\left(P_{k}\right)\right\rangle .
$$

This is a topological invariant in the sense that it is unchanged under continuous changes in the metric of $\Sigma$ and the metric and complex structure of $M$ (but possibly depends on the homotopy type of the almost complex structure of $M$ ). The invariance of (3.11) can be deduced from standard arguments that were repeatedly used in [4]. Under an infinitesimal change in the metric and complex structure of $M,(3.11)$ changes by

$$
\delta Z=\left\langle\mathcal{O}_{A_{1}}^{(0)}\left(P_{1}\right) \cdots \mathcal{O}_{A_{k}}^{(0)}\left(P_{k}\right) \cdot(-\delta \mathscr{L})\right\rangle,
$$

with $\delta \mathscr{L}$ the change in $\mathscr{L}$ due to the change in metric and complex structure of $\Sigma$ or $M$. But we have observed that $\mathscr{L}=\{Q, V\}$ for some $V$. So

$$
\partial Z=\left\langle\prod_{i} \mathcal{O}_{A_{i}}^{(0)}\left(P_{i}\right) \cdot\{Q, V\}\right\rangle=-\left\langle\left\{Q, \prod_{i} \mathcal{O}_{A_{i}}^{(0)}\left(P_{i}\right) \cdot V\right\}\right\rangle=0
$$

This has some interesting consequences. Being independent of the metric on $\Sigma, Z$ must be independent of the choice of distinct points $P_{i}$. But it is very illuminating to check this explicitly. Viewing $\mathbb{C}_{A}^{(0)}(P)$ as an operator valued $\mathcal{O}$-form on $\Sigma$, we compute its exterior derivative

$$
\begin{aligned}
\frac{\partial}{\partial \sigma^{\alpha}} \mathcal{O}_{A}^{(0)} & =\frac{\partial}{\partial \sigma^{\alpha}}\left(A_{i_{1} \ldots i_{n}} \chi^{i_{1}} \chi^{i_{2}} \cdots \chi^{i_{n}}\right) \\
& =\partial_{i_{0}} A_{i_{1} i_{2} \ldots i_{n}} \frac{\partial u^{i_{0}}}{\partial \sigma^{\alpha}} \chi^{i_{1}} \cdots \chi^{i_{n}}+n A_{i_{1} i_{2} \ldots i_{n}} \frac{D \chi^{i_{1}}}{D \sigma^{\alpha}} \chi^{i_{2}} \cdots \chi^{i_{n}}
\end{aligned}
$$

\footnotetext{
7 This is essentially the structure that was conjectured in [16] as the $1+1$ dimensional analogue of Donaldson polynomials. If one chooses $A$ to be the Poincaré dual of a hypersurface $Y$ in $M$, then $\mathscr{C}_{A}(P)$ is zero except for maps $\Sigma \rightarrow M$ that map $P$ into $Y$. This corresponds to the structure sketched in $[16]$
} 
If $d A=0$, then (3.14) can be rewritten

where

$$
d \mathcal{O}_{A}^{(0)}=i\left\{Q, \mathcal{O}_{A}^{(1)}\right\},
$$

$$
\mathcal{O}_{A}^{(1)}=\operatorname{in} A_{i_{1} i_{2} \ldots i_{n}} \cdot d u^{i_{n}} \chi^{i_{2}} \cdots \chi^{i_{n}}
$$

is an operator valued one form on $\Sigma$. Equation (3.15) shows that if $P$ and $P^{\prime}$ are any two points on $\Sigma$ then the difference $\mathcal{O}_{A}^{(0)}(P)-\mathcal{O}_{A}^{(0)}\left(P^{\prime}\right)$ is a BRST commutator. Indeed, picking any path $\gamma$ from $P^{\prime}$ to $P$,

$$
\mathcal{O}_{A}^{(0)}(P)-\mathcal{O}_{A}^{(0)}\left(P^{\prime}\right)=\left\{Q, \int_{\gamma} \mathcal{O}_{A}^{(1)}\right\} .
$$

Equation (3.17) implies that (3.11) is independent of the $P_{i}$, as long as the differential forms $A_{i}$ are all closed. For

$$
\left\langle\left(\mathcal{O}_{A}^{(0)}(P)-\mathcal{O}_{A}^{(0)}\left(P^{\prime}\right)\right) \cdot \Pi \mathcal{O}_{A_{l}}^{(0)}\left(P_{i}\right)\right\rangle=\left\langle\left\{Q,\left(\int_{\gamma} \mathcal{O}_{A_{i}}^{(1)} \cdot \Pi \mathcal{O}_{A}^{(0)}\left(P_{i}\right)\right)\right\}\right\rangle=0 .
$$

At this point, however, we can use (3.15) to define new BRST invariant observables. For let $\gamma$ be a one dimensional homology cycle on $\Sigma$. Let

$$
W_{A}(\gamma)=\int_{\gamma} \mathcal{O}_{A}{ }^{(1)}
$$

Then

$$
\left\{Q, W_{A}(\gamma)\right\}=\int_{\gamma}\left\{Q, \mathcal{O}_{A}{ }^{(1)}\right\}=-i \int_{\gamma} d \mathcal{O}_{A}{ }^{(0)}=0 .
$$

Therefore, $W_{A}(\gamma)$ is a new BRST invariant operator that can be considered in correlation functions. Let us now see that $W(\gamma)$ depends only on the homology class for $\gamma$. For if $\gamma=\partial \beta$ for some two chain $\beta$, then

$$
\int_{\gamma} \mathcal{O}_{A}^{(1)}=\int_{\beta} d \mathcal{O}_{A}^{(1)} \text {. }
$$

But a little calculation shows that

where

$$
\begin{aligned}
d \mathcal{O}_{A}{ }^{(1)}= & i n \partial_{i_{0}} A_{i_{1} i_{2} \ldots i_{n}} \cdot d u^{i_{0}} \wedge d u^{i_{1}} \cdot \chi^{i_{2}} \cdots \chi^{i_{n}} \\
& +i n(n-1) A_{i_{1} i_{2} \ldots i_{n}} d u^{i_{1}} \wedge D \chi^{i_{2}} \chi^{i_{3}} \cdots \chi^{i_{n}} \\
= & i\left\{Q, \mathcal{O}_{A}{ }^{(2)}\right\},
\end{aligned}
$$

$$
\mathcal{O}_{A}{ }^{(2)}=-\frac{n(n-1)}{2} A_{i_{1} i_{2} \ldots i_{n}} d u^{i_{1}} \wedge d u^{i_{2}} \chi^{i_{3}} \cdots \chi^{i_{n}} \text {. }
$$

Hence if $\gamma=\partial \beta$, then

$$
W_{A}(\gamma)=\int_{\gamma} \mathcal{O}_{A}^{(1)}=\int_{\beta} d \mathcal{O}_{A}^{(1)}=i\left\{Q, \int_{\beta} \mathcal{O}_{A}^{(2)}\right\}
$$

is trivial in the BRST sense.

At the same time (3.22) leads to a new BRST invariant integral, namely

$$
W_{A}(\Sigma)=\int_{\Sigma} \mathcal{O}_{A}^{(2)}
$$


For

$$
\left\{Q, W_{A}(\Sigma)\right\}=\int_{\Sigma}\left\{Q, \mathcal{O}_{A}^{(2)}\right\}=-i \int_{\Sigma} d \mathcal{O}_{A}^{(1)}=0 .
$$

Let us state these results in a more unified way. For any closed differential form $A$ on $M$, we have defined the three operators $\mathcal{O}_{A}{ }^{(0)}, \mathcal{O}_{A}{ }^{(1)}$, and $\mathcal{O}_{A}{ }^{(2)}$, obeying

$$
\begin{aligned}
0 & =i\left\{Q, \mathcal{O}_{A}{ }^{(0)}\right\}, & d \mathcal{O}_{A}{ }^{(0)}=i\left\{Q, \mathcal{O}_{A}{ }^{(1)}\right\}, \\
d \mathcal{O}_{A}{ }^{(1)} & =i\left\{Q, \mathcal{O}_{A}{ }^{(2)}\right\}, & d \mathcal{O}_{A}{ }^{(2)}=0 .
\end{aligned}
$$

For $A$ an $n$-form and $k=0,1,2$, it is clear that $\mathcal{O}_{A}{ }^{(k)}$ has $U=n-k$. The equations (3.27) imply that if $\gamma$ is a homology $k$-cycle on $\Sigma$, then

$$
W_{A}(\gamma)=\int_{\gamma} \mathcal{O}_{A}{ }^{(k)}
$$

is BRST invariant, and-up to a BRST commutator-depends only on the homology class of $\gamma$.

The interesting observables may then be described as follows. Pick closed forms $A_{1}, A_{2}, \ldots, A_{k}$ of degrees $d_{1}, \ldots d_{k}$ on $M$, and homology cycles $\gamma_{1}, \ldots, \gamma_{k}$ of dimensions $t_{1}, \ldots, t_{k}$. Let $W_{A_{i}}\left(\gamma_{i}\right)=\int_{\gamma_{i}} \mathcal{O}_{A_{t}}{ }^{\left({ }_{t}\right)}$. Then the BRST-invariant observables are

$$
Z\left(\left(A_{1}, \gamma_{1}\right), \ldots,\left(A_{k}, \gamma_{k}\right)\right)=\left\langle\prod_{i} W_{A_{l}}\left(\gamma_{i}\right)\right\rangle .
$$

These observables, moreover, are topological invariants (invariant under continuous deformations of the metric and complex structure of $\Sigma$ and $M$ ), by an argument that was sketched above.

3.2 Evaluation of the Invariants. It remains to give a prescription for evaluating the invariants that we have just described, and to show that they are non-trivial and have interesting geometrical consequences.

First of all, since the quantities of interest are independent of the metric of $M$, we can consider a one parameter family of metrics of the form $g_{i j}^{(t)}=t \cdot g_{i j}$, with $g_{i j}$ some given metric. This has the effect of substituting $\mathscr{L} \rightarrow t \mathscr{L}$. For large $t$ the Feynman path integral

$$
\langle\mathcal{O}\rangle=\int \mathscr{D} X e^{-t \mathscr{L}} \cdot \mathcal{O},
$$

is dominated by the minima of $\mathscr{L}$. Looking back to (2.16), we see that the bosonic terms in the Lagrangian are positive semi-definite, and vanish if and only if

$$
\partial_{\alpha} u^{i}+\varepsilon_{\alpha \beta} J_{j}^{i} \partial^{\beta} u^{j}=0
$$

This is the equation for a holomorphic map $\phi: \Sigma \rightarrow M$; we will call such maps instantons.

Of course, in defining the path integral (3.30), we have had to pick a topological class of maps of $\Sigma$ to $M$. Whether instantons exist will depend on the chosen topological class. If for some topological class there actually is an instanton, one naturally wishes to know if it is isolated of forms part of a family. Given a solution of (3.31), the requirement that a slightly displaced map, with $u^{i}$ replaced by $u^{i}+\partial u^{i}$, 
should obey (3.31) to lowest order in $\delta u^{i}$ is

$$
0=D_{\alpha} \delta u^{i}+\varepsilon_{\alpha \beta} J_{j}{ }_{j} D^{\beta} \delta u^{j}+\varepsilon_{\alpha \beta} D_{k} J^{i}{ }_{j} \partial^{\beta} u^{j} \partial u^{k} .
$$

We write this as $0=\bar{D} \delta u, \bar{D}$ being the linear operator that appears in (3.32). The zero modes of $\bar{D}$ correspond to infinitesimal deformations of an instanton.

Given an infinitesimal deformation of an instanton, there may be an obstruction to integrating it to give a finite deformation of the instanton. It is well known (but will not be demonstrated here), that the obstruction lies in the cokernel of $\bar{D}$ (or equivalently, the kernel of the adjoint operator $\bar{D}^{*}$ ). Let $\mathscr{M}$ denote the moduli space of instantons of a given topological type. Formally, one expects that the dimension of $\mathscr{M}$ should equal the number of infinitesimal deformations of the instanton minus the number of obstructions to integrating such infinitesimal deformations; this is precisely the index of $\bar{D}$. (For a topological class of maps of $\Sigma$ to $M$ which is such that the index is negative, instantons will typically not exist.) Mathematically, the kernel and cokernel of $\bar{D}$ correspond to $H^{0}\left(\Sigma, \phi^{*}(T)\right)$ and $H^{1}\left(\Sigma, \phi^{*}(T)\right)$, with $\phi^{*}(T)$ denoting the pullback of the tangent bundle $T$ of $M$ via the map $\phi: \Sigma \rightarrow M$. The index of $\bar{M}$ is the same as the dimension of $H^{0}$ minus the dimension of $H^{1}$.

Looking back to the Lagrangian (2.16) (and remembering the kinematic condition obeyed by $\rho$ ), we see that in expanding around an instanton solution, the zero modes of $\chi$ and $\rho$ correspond exactly to the kernel and cokernel of $\bar{D}$. In quantum field theory, the number of $\chi$ zero modes minus the number of $\rho$ zero modes (or in other words, the index of $\bar{D}$, which we will denote as $n$ ) is a very important quantity. Recall that at the classical level (2.16) has a conserved quantum number $U$ with the values +1 and -1 for $\chi$ and $\rho$, respectively. As in the solution [17] of the $U(1)$ problem in QCD, the number of $\chi$ zero modes minus the number of $\rho$ zero modes is the violation of $U$ by the instanton. In other words, the integration measure in (3.30) is not invariant under $U$ but is homogeneous of degree $-n$; and, if $\mathcal{O}$ is decomposed in components with a definite $U$ quantum number, a non-zero contribution comes only from the component of degree $+n$. Our basic invariant

$$
Z\left(\left(A_{1}, \gamma_{1}\right), \ldots\left(A_{k}, \gamma_{k}\right)\right)=\left\langle\prod_{i} W_{A_{i}}\left(\gamma_{i}\right)\right\rangle
$$

associated with closed forms $A_{i}$ on $M$ of degree $d_{i}$ and homology cycles $\gamma_{i}$ on $\Sigma$ of degree $t_{i}$ is homogeneous under $U$ of degree

$$
\Delta U=\sum\left(d_{i}-t_{i}\right)
$$

It will vanish unless one chooses a homotopy class of maps of $\Sigma$ to $M$ such that $n=\Delta U$.

We will here restrict ourselves to the case in which $H^{1}=0$, that is, to the case in which there are no $\rho$ zero modes and the $\chi$ zero modes are precisely the tangents to the moduli space $\mathscr{M}$ of instantons. This situation (which is realized in many interesting examples, some of which will be noted later) is very similar to the situation considered in [4]. In carrying out the Feynman path integral (3.30), one can ignore the non-zero modes; for in leading order of perturbation theory, which 
is adequate for topological purposes, the non-zero modes give rise to boson and fermion determinants which cancel because of the fermionic symmetry. Everything thus reduces to an integral over the instanton moduli space.

Perhaps it is useful to distinguish between the underlying bosonic moduli space $\mathscr{M}$ of holomorphic maps $\Sigma \rightarrow M$ (of a given topological type), and the "moduli superspace," $\hat{\mathscr{M}}$, which includes the fermionic zero modes as coordinates as well. This extended moduli space may locally be described with bosonic coordinates $a^{\lambda}, \lambda=1 \ldots n$ and fermionic coordinates $\chi^{\lambda}$ which transform like $d a^{\lambda}$. To be very explicit about this, for future use, the $a^{\lambda}$ parametrize a family of maps of $\Sigma$ to $M$, which we may describe by saying that a point $P \in \Sigma$ is mapped

$$
P \rightarrow u^{k}\left(P ; a^{\lambda}\right) \text {. }
$$

Here we have indicated explicitly the dependence on the $a^{\lambda}$. Equation (3.35) says that the $u^{k}(P)$ (and more generally any functionals of the $u^{k}(P)$ ) can be regarded, through their dependence on the $a^{\lambda}$, as functions on instanton moduli space. This can be extended to include the odd coordinates on moduli space. Pick a basis of solutions of (3.32), say $\delta u_{(\lambda)}^{i}, \lambda=1 \ldots n$, corresponding to a basis of the tangent space of $\mathscr{M}$. Any solution $\chi^{i}(P)$ of (3.32) has an expansion

$$
\chi^{i}(P)=\sum_{\lambda=1}^{n} \chi^{\lambda} \delta u^{i}\left(P ; a^{\tau}\right)(\lambda)
$$

with constant (anticommuting) coefficients $\chi^{\lambda}$. We regard the $\chi^{\lambda}$ as the odd coordinates, of moduli space, and (3.36) is the formula expressing the $\chi^{i}(P)$, after restriction to moduli space, as functions of the even and odd coordinates $a^{\lambda}$ and $\chi^{\lambda}$ of moduli space.

The next important point is that there is a canonical measure on instanton moduli space,

$$
d \mu=d a^{1} d a^{2} \cdots d a^{n} d \chi^{1} d \chi^{2} \cdots d \chi^{n},
$$

since $d a^{\lambda}$ and $d \chi^{\lambda}$ transform oppositely under any reparametrization of $\mathscr{M}$. A function on $\hat{\mathscr{M}}$ which is homogeneous of degree $k$ under $U$ is of the general form

$$
\Phi=W_{\lambda_{1} \lambda_{2} \ldots \lambda_{k}} \chi^{\lambda_{1}} \chi^{\lambda_{2}} \cdots \chi^{\lambda_{k}},
$$

and corresponds to a differential form $\hat{\Phi}=W_{\lambda_{1} \lambda_{2} \cdots \lambda_{k}} d a^{\lambda_{1}} d a^{\lambda_{2}} \cdots d a^{\lambda_{k}}$ of order $k$ on $\hat{\mathscr{M}}$. Integration of functions over $\hat{\mathscr{M}}$ is the same as integration of differential forms over $\mathscr{M}$ :

$$
\int_{\mathscr{M}} \Phi=\int_{\mathscr{M}} \hat{\Phi}
$$

Of course, (3.39) vanishes except for $k=n$.

Now, we want to give a recipe for evaluating the topological invariants (3.29):

$$
Z\left(\left(A_{1}, \gamma_{1}\right), \ldots\left(A_{k}, \gamma_{k}\right)\right)=\left\langle\prod_{i} W_{A_{t}}\left(\gamma_{i}\right)\right\rangle
$$

We recall that the $A_{i}$ are closed differential forms on $M$ of degrees $d_{i}$, the $\gamma_{i}$ are homology cycles on $M$ of degrees $t_{i}$, and

$$
W_{A_{i}}\left(\gamma_{i}\right)=\int_{\gamma_{i}} \mathcal{O}_{A_{i}}^{\left(t_{2}\right)}
$$


The recipe for evaluating (3.40) to lowest order in perturbation theory is as follows. ${ }^{8}$ Expanding around the minima of the classical action, the operation $\langle>$ reduces to an integral over $\hat{\mathscr{M}}$. Each $W_{A_{i}}\left(\gamma_{i}\right)$ becomes a function $\Phi\left(A_{i}, \gamma_{i}\right)$ on $\hat{\mathscr{M}}$ of degree $d_{i}-t_{i}$ (corresponding to a differential form $\hat{\Phi}$ of the same degree on $\mathscr{M}$ ). Then (3.40) reduces to

$$
Z\left(\left(A_{1}, \gamma_{1}\right), \ldots\left(A_{k}, \gamma_{k}\right)\right)=\int_{\|} \Phi\left(A_{1}, \gamma_{1}\right) \ldots \Phi\left(A_{k}, \gamma_{k}\right)
$$

At that remains, therefore, is to explain cocretely how to interpret the $W_{A_{l}, \gamma_{t}}$ as differential forms on $\mathscr{M}$ or functions on $\hat{\mathscr{M}}$. The general procedure in quantum field theory is that one should "integrate out the non-zero modes" and express the operators

$$
W_{A_{1}}\left(\gamma_{i}\right)=\int_{\gamma_{1}} \mathcal{O}_{A_{1}}^{\left(t_{1}\right)}
$$

in terms of the zero modes only. In [4] this involved a slightly non-trivial step of integrating out certain degrees of freedom, but in the case at hand (as long as $H^{1}=0$ ), life is simpler. The $\mathcal{O}_{A_{t}}^{\left(t_{2}\right)}$ are defined as functionals of arbitrary $u, \chi$, and $\rho$. It makes sense to simply restrict them to instanton moduli space and interpret them as functions on it. For instance, if $\gamma$ is a zero cycle, simply a point $P \in \Sigma$, then we have defined for any differential form $A$ on $M$ the operator

$$
\mathcal{O}_{A}^{(0)}(P)=A_{i_{1} i_{2} \ldots i_{n}}\left(u^{k}\left(P ; a^{\lambda}\right)\right) \chi^{i_{1}}(P) \chi^{i_{2}}(P) \cdots \chi^{i_{k}}(P) .
$$

Here $u^{k}\left(P ; a^{\lambda}\right)$ denotes the image of the point $P \in \Sigma$ under the map $\Sigma \rightarrow M$ described by the $u^{k}$, and as in (3.35) their dependence on the parameters of instanton moduli space is being indicated explicitly. Likewise, the $\chi^{i}(P)$ may as in (3.36) be regarded as functions on instanton moduli space. So, as in $(3.35)$ and $(3.36), O_{A}{ }^{(0)}(P)$, or any other functional of the $u^{j}$ and $\chi^{k}$, may be restricted to give a functional on the even and odd coordinates of instanton moduli space $\hat{\mathscr{M}}$. In the case $H^{1}=0$ to which we have restricted ourselves, this is all that is required to determine the functions $\Phi\left(A_{j}, \gamma_{j}\right)$ that appear in (3.42).

It is possible to give a more straightforward and classical account of this, more in the spirit of Donaldson's description of his four dimensional invariants. An instanton corresponds to a map $\alpha: \Sigma \rightarrow M$. If we are given a family of instantons, parametrized by a parameter space $T$, we get a map $\tilde{\alpha}: \Sigma \times T \rightarrow M$. If we take $T$ to be the moduli space of instantons of a given topological class, we get the "universal instanton" of that class, which is a map

$$
\hat{\alpha}: \Sigma \times \mathscr{M} \rightarrow M \text {. }
$$

Suppose we are given a closed differential form $A$ on $M$ of degree $d$. Then its pullback to $\Sigma \times \mathscr{M}$

$$
\hat{A}=\hat{\alpha}^{*}(A)
$$

is a closed $d$-form on $\Sigma \times \mathscr{M}$. If we are given a homology cycle $\gamma$ on $M$, of dimension

8 A somewhat more detailed explanation was given for similar steps in [4] 
$t$, then by restricting $\hat{A}$ to $\gamma \times \mathscr{M}$, and then integrating over $\gamma$, we get a closed $d-t$ form

$$
\hat{\Phi}(A, \gamma)=\int_{\gamma} \hat{A}
$$

on $\mathscr{M}$. The invariants defined in (3.40) are then simply

$$
Z\left(\left(A_{1}, \gamma_{1}\right), \ldots\left(A_{k}, \gamma_{k}\right)\right)=\int_{\widehat{\mu}} \widehat{\Phi}\left(A_{1}, \gamma_{1}\right) \wedge \hat{\Phi}\left(A_{2}, \gamma_{2}\right) \wedge \cdots \wedge \hat{\Phi}\left(A_{k}, \gamma_{k}\right)
$$

Alternatively, these invariants may be written as an integral over $\hat{\mathscr{M}}$,

$$
Z\left(\left(A_{1}, \gamma_{1}\right), \ldots\left(A_{k}, \gamma_{k}\right)\right)=\int_{\hat{M}} \Phi\left(A_{1}, \gamma_{1}\right) \cdot \Phi\left(A_{2}, \gamma_{2}\right) \cdots \Phi\left(A_{k}, \gamma_{k}\right)
$$

with $\Phi(A, \gamma)$ being the function on $\hat{\mathscr{M}}$ that corresponds to the differential form $\hat{\Phi}(A, \gamma)$ on $\mathscr{M}$.

The key formulas such as (3.47) and (3.48) in the last paragraph are so elementary that one may well ask what is the virtue in the quantum field theory viewpoint. One answer, of course, is that one would like to prove that (3.48) is a topological invariant, that is, unchanged in continuous variations of the metric and complex structure of $\Sigma$ and $M$. The BRST invariant quantum field theory gives a recipe for proving this, using the fact that the change in (2.16) under an infinitesimal change in the metric and almost complex structure of $\Sigma$ and $M$ is a BRST commutator. The recipe was detailed in Sect. 5 of [4] and will not be repeated to here.

3.3 An Application. Here I will briefly indicate that the discussion in the last section is not vacuous by showing that the invariants in (3.40) actually have interesting geometrical consequences. In fact, we will see that results along the lines of Gromov's work [5] are natural in this framework.

To obtain some non-trivial results, we must show in particular that the invariants defined in (3.40) are not identically zero. Donaldson, for example, showed that his polynomial invariants for four manifolds were non-zero by showing that they were positive definite for algebraic surfaces, under certain conditions. This depended on the positivity of the intersections of algebraic cycles on an algebraic variety. It is natural to similarly try to show that our invariants

$$
Z\left(\left(A_{1}, \gamma_{1}\right), \ldots\left(A_{k}, \gamma_{k}\right)\right)=\left\langle\prod_{i=1}^{k} W\left(A_{i}, \gamma_{i}\right)\right\rangle
$$

are not identically zero by using appropriate positivity properties associated with Kahler geometry.

So we now specialize to the case in which $M=K$ is a Kahler manifold, the Kahler form being a $(1,1)$ form $\omega$. The Kahler form has the following fundamental positivity property. Let $V$ by any $(1,0)$ vector field on $K$, and let $\bar{V}$ be its complex conjugate, which is type $(0,1)$. Then

$$
i \omega(V, \bar{V}) \geqq 0,
$$

and moreover (3.51) vanishes only where $V=0$. 
Pick a Riemann surface $\Sigma$, and a homotopy class of maps of $\Sigma$ to $K$ which is such that the moduli space of holomorphic maps $\Sigma \rightarrow K$ of this topological type is non-empty. This moduli space is, of course, naturally a Kahler manifold. Assume, moreover, that in the moduli problem, expanding around such a holomorphic map, one finds $H^{1}=0$. We will prove that (3.50) is strictly positive in the following special case. Let the homology cycles $\gamma_{i}$ be points $P_{1}, \ldots P_{k}$. And let the differential forms $A_{i}$ all equal the Kahler form $\omega$. Then the invariant whose positivity we wish to establish is

$$
Z\left(P_{1}, P_{2} \cdots P_{k}\right)=\left\langle\mathcal{O}_{\omega}^{(0)}\left(P_{1}\right) \cdots \mathcal{O}_{\omega}^{(0)}\left(P_{k}\right)\right\rangle,
$$

where $k$ is the complex dimension of the instanton moduli space. Because we assume that $H^{1}=0$, the evaluation of (3.52) reduces to the simplified formula (3.48). This may be described very explicitly as follows. Let $\hat{\alpha}: \Sigma \times \mathscr{M} \rightarrow K$ be the "universal instanton." For any $P \in \Sigma, \hat{\alpha}$ restricted to $P \times \mathscr{M}$ gives a holomorphic map $\alpha_{P}: \mathscr{M} \rightarrow K$. Let $\omega_{P}$ be the differential form on $\mathscr{M}$ defined by

$$
\omega_{P}=\alpha_{P}^{*}(\omega) \text {. }
$$

The $\omega_{P}$ are $(1,1)$ forms on $\mathscr{M}$. Equation (3.52) then can be written

$$
Z\left(P_{1}, \ldots P_{k}\right)=\int_{\mathscr{M}} \omega_{P_{1}} \wedge \cdots \wedge \omega_{P_{k}} .
$$

We wish to show that this is positive. In fact, positivity of (3.54) is a consequence of (3.51) plus the Riemann-Roch theorem. Let $S$ be a point in $\mathscr{M}$, and let $X$ be a tangent vector of type $(1,0)$ to $\mathscr{M}$ at $S$. Concretely, $S$ corresponds to a holomorphic map $\phi: \Sigma \rightarrow K$, and $X$ corresponds to an element $\lambda$ of $H^{0}\left(\Sigma, \phi^{*}(T)\right)$, with $T$ the holomorphic tangent bundle of $K$. Restricted to $P \in \Sigma$, $\lambda$ gives a $(1,0)$ tangent vector $V$ at $\phi(P)$, and we have

$$
i \omega_{P}(X, \bar{X})=i \omega(V, \bar{V}) .
$$

Therefore, (3.51) implies that $i \omega_{P}(X, \bar{X}) \geqq 0$ for all $X$. Moreover, we can interpret the kernel of $\omega_{P}$ (that is, the space of $X$ for which (3.55) vanishes). It consists of infinitesimal displacements of the instanton $\phi: \Sigma \rightarrow K$ that leave fixed the image of the point $P \in \Sigma$. If $\mathcal{O}(-P)$ is the line bundle on $\Sigma$ with divisor $-P$ (the function 1 has a pole at $P$ when regarded as a section of $\mathcal{O}(-P))$, then the kernel of $\omega_{P}$ is $H^{0}\left(\Sigma, \phi^{*}(T) \otimes \mathcal{O}(-P)\right)$.

In (3.54), we are considering the wedge product on a Kahler manifold of complex dimension $k$ of $k(1,1)$ forms, each of which is positive semi-definite. Therefore, the measure which is being integrated in (3.54) is positive semi-definite. To show that (3.54) is positive, it is enough to show that the measure in (3.54) is not identically zero. The measure in (3.54) vanishes at a point $S \in \mathscr{M}$ if and only if the intersection of the kernels of the $\omega_{P_{2}}$ is non-zero. The intersections of the kernels of the $\omega_{P_{2}}$ is non-zero if and only if there is an element of $H^{0}\left(\Sigma, \phi^{*}(T)\right)$ which vanishes at each of the $P_{i}$. Since (3.54) is independent of the choices of the points $P_{i}$, we are entitled to make convenient choices of these points. Any generic choice of the $P_{i}$ will suffice to show that the measure is not identically zero. For any generic set of $n$ points in $\Sigma$ has the property that of the $n$ linearly independent 
sections of $\phi^{*}(T)$, there is no linear combination that vanishes at each of these $n$ points. Consequently, (3.54) is positive.

Before giving some geometrical consequence, let us mention some simple examples in which the above hypotheses are satisfied. One example (extensively discussed by Gromov) is the case in which $\Sigma$ is of genus zero, and $K$ is $C P^{n}$ with its standard complex structure. We consider degree one maps $\Sigma \rightarrow K$; the holomorphic curves of this topological type are a family of complex dimension $2 n+1$. For a second example, let $K^{\prime}$ be a cubic surface in $C P^{3}$. A generic hyperplane section of $K^{\prime}$ is then a curve of genus one, which we will call $\Sigma^{\prime}$. This gives a Riemann surface $\Sigma^{\prime}$ with a holomorphic embedding $\phi: \Sigma^{\prime} \rightarrow K^{\prime}$. It is easy to see that this is one point on a three complex dimensional moduli space of holomorphic maps of $\Sigma^{\prime}$ to $K^{\prime}$. (This hyperplane sections of $K^{\prime}$ are a three parameter subfamily. A two parameter subfamily of these have the same complex structure as $\Sigma^{\prime}$. Since we are studying not subvarieties of $K^{\prime}$ but maps $\Sigma^{\prime} \rightarrow K^{\prime}$, we must add one parameter to take account of holomorphic isomorphisms of $\Sigma^{\prime}$ with itself as these can be composed with any given embedding in $K^{\prime}$.) In each of these two examples, it is easy to see that $H^{1}\left(\Sigma, \phi^{*}(T)\right)=0$, so our arguments apply, and (3.54) is positive.

What of a non-trivial nature can we learn this way? Consider deforming the complex structure of $K$ or $K^{\prime}$ to a not necessarily integrable almost complex structure of the same homotopy type. Since (3.54) is nonzero, and is invariant under the deformation of complex structure, it must remain non-zero for the deformed problem. On the other hand, (3.54) can be evaluated by integration over the moduli space of holomorphic curves. So we conclude for any chosen almost complex structure on $K$ or $K^{\prime}$ (of the same homotopy type), there must be a $2 n+1$ complex dimensional family of holomorphic maps $\Sigma \rightarrow K$, and a three complex dimensional family of holomorphic maps $\Sigma^{\prime} \rightarrow K^{\prime}$, giving the same value of (3.54) as in the undeformed problem. Statements such as this, along with more refined versions, some of which can be obtained by reasoning along the lines just indicated, were established by Gromov.

\section{Topological Gravity in Two Dimensions}

So far in this paper, the two dimensional metric has played a purely passive role. The model we have considered has possessed its fermionic symmetry on an arbitrary Riemann surface $\Sigma$ with any metric. However, the metric has not been a dynamical field; it has simply been prescribed and fixed as part of the definition of the problem. In particular, the metric is invariant under the fermionic symmetry of the model of Eq. (2.16). Were the metric not invariant, we would indeed not regard that transformation as a symmetry, since a different metric would in the context of our discussion until this point correspond to a different model.

In this section, we will change the rules and consider the coupling of the topological sigma model that we have already formulated to two dimensional gravity. Of course, we wish to carry out this coupling in a way that preserves the global fermionic symmetry $Q$, of square zero, that is responsible for most of the 
Table II. The conformal dimensions, global charges, and statistics of the various fields

\begin{tabular}{lrrl}
\hline & $D$ & $U$ & Statistics \\
\hline$e_{\alpha a}$ & -1 & 0 & + \\
$\psi_{a b}$ & 0 & 1 & - \\
$C_{\alpha}$ & -1 & 2 & + \\
$R_{a}$ & $k$ & -2 & + \\
$\xi_{a}$ & $k$ & -1 & - \\
\hline
\end{tabular}

interesting features of our earlier discussion. The first step-even before coupling two dimensional gravity to the sigma model - is to formulate a purely gravitational model possessing an appropriate fermionic symmetry. This will then be a two dimensional analogue of the four dimensional model of "topological gravity" formulated in [18]. ${ }^{9}$

We will describe two dimensional gravity in terms of a basis $e^{a}, a=1,2$ of orthonormal one forms. In components $e^{a}=e_{\alpha}^{a} d \sigma^{\alpha}, \sigma^{\alpha}$ being local coordinates on $\Sigma$. The two dimensional metric tensor is $h_{\alpha \beta}=e_{\alpha}{ }^{a} e_{\beta a}$. "Lorentz" indices $a, b, c$ are raised and lowered with the Kronecker metric $\delta_{a b}$; "world" indices $\alpha, \beta, \gamma$ are raised and lowered with the metric $h_{\alpha \beta}$. The inverse of $h_{\alpha \beta}$ is denoted $h^{\alpha \beta} . e^{\alpha a}=h^{\alpha \beta} e_{\beta}{ }^{a}$ is the inverse of $e_{\beta}{ }^{a}$; that is, $e^{\alpha a} e_{\beta a}=\delta^{\alpha}{ }_{\beta}, e^{\alpha a} e_{\alpha b}=\delta^{a}{ }_{b}$. A tangent vector $V=V^{\alpha}\left(\partial / \partial \sigma^{\alpha}\right)$ is conveniently described by its components $V^{a}=e^{a}{ }_{\alpha} V^{\alpha}$ in the basis $e^{a}{ }_{\alpha}$. The covariant derivative of a vector field so described is

$$
D_{\alpha} V^{a}=\partial_{\alpha} V^{a}+\omega_{\alpha} \cdot \varepsilon^{a b} V_{b},
$$

where $\varepsilon_{a b}=-\varepsilon_{b a}, \varepsilon^{a b} \varepsilon_{c b}=\delta^{a}{ }_{c}$, and $\omega_{\alpha}$ is the Levi-Cevita connection, determined by requiring that

$$
D_{\alpha} e_{\beta}^{a}=0
$$

In addition to the vierbein $e_{\alpha}^{a}$, we introduce certain other matter fields indicated in the table. For the fields $e_{\alpha a}, \psi_{a b}$, and $C_{a}$, we postulate the same transformation law as in [18]:

$$
\begin{aligned}
\delta e_{\alpha a} & =i \varepsilon e_{\alpha}^{b} \psi_{a b}, \\
\delta \psi_{a b} & =\frac{\varepsilon}{2}\left(D_{a} C_{b}+D_{b} C_{a}-\delta_{a b} D_{f} C^{f}\right), \\
\delta C_{a} & =i \varepsilon \psi_{a b} C^{b} .
\end{aligned}
$$

\footnotetext{
${ }^{9}$ In fact, some interesting two dimensional models can be obtained by dimensional reduction of that four dimensional model. These would apparently correspond to $R^{2}$ gravity rather than the Einstein gravity that we will aim for here. However, the relation with the four dimensional construction makes the construction here rather easy
} 
Here $D_{a}$ is an abbreviation for $e_{a}{ }^{\alpha} D_{\alpha}$. Equation (4.3) implies the transformation laws

$$
\delta e^{\alpha a}=-i \varepsilon e_{b}^{\alpha} \psi^{a b}, \quad \delta \omega_{\alpha}=-i \varepsilon e_{\alpha b} e^{\gamma a} \varepsilon_{a c} D_{\gamma} \psi^{b c}
$$

for the inverse vierbein and the connection.

Trying to close the algebra, one finds

$$
\left(\delta_{\eta} \delta_{\varepsilon}-\delta_{\varepsilon} \delta_{\eta}\right) e_{\alpha a}=D_{\alpha}\left(-2 i \eta \varepsilon C_{a}\right)+\eta \varepsilon \cdot \varepsilon_{a b} \Lambda e_{\alpha}^{b}+i \eta \varepsilon \cdot S \cdot e_{\alpha a},
$$

with

$$
\Lambda=\left(-\varepsilon^{a b} \psi_{a x} \psi_{b}{ }^{x}-i \varepsilon^{a b} D_{a} C_{b}\right), \quad S=D_{f} C^{f} .
$$

The three terms on the right-hand side of (4.6) are to be interpreted respectively as the change in $e_{\alpha}{ }^{a}$ under an infinitesimal diffeomorphism generated by the vector field $-2 i \eta \varepsilon C^{\alpha}$, a local Lorentz transformation with parameter $\eta \varepsilon \Lambda$, and a Weyl rescaling with parameter $-i \eta \varepsilon S$. (Recall that $e_{\alpha a}$ has conformal dimension -1 ). The algebra closes on the other fields with the same parameters:

$$
\begin{gathered}
\left(\delta_{\eta} \delta_{\varepsilon}-\delta_{\varepsilon} \delta_{\eta}\right) C_{a}=-2 i \eta \varepsilon C^{\alpha} D_{\alpha} C_{a}+\eta \varepsilon \Lambda \varepsilon_{a b} C^{b}+i \eta \varepsilon S C_{a}, \\
\left(\delta_{\eta} \delta_{\varepsilon}-\delta_{\varepsilon} \delta_{\eta}\right) \psi_{a b}=-2 i \eta \varepsilon C^{\alpha} D_{a} \psi_{a b}+\eta \varepsilon \Lambda\left(\varepsilon_{a}{ }^{x} \psi_{x b}+\varepsilon_{b}{ }^{x} \psi_{a x}\right) .
\end{gathered}
$$

A term $D_{\psi} \cdot S \cdot \psi_{a b}$ is absent from the right-hand side of the second equation in (4.7), since the conformal dimension $D_{\psi}$ of $\psi$ is zero.

For coupling of sigma models to dynamical gravity, the above formulas are all that we will need; that is, as we will see in the next subsection, BRST invariant sigma models can be coupled to the $e, \psi, C$ multiplet while preserving the fermionic symmetries. We would like, however, to conclude this subsection with some comments on the attempt to write an action for the purely gravitational multiplet - though our discussion of this point will be neither complete nor entirely satisfactory.

Though $e, \psi$, and $C$ form a closed multiplet with the above transformation laws, it is not possible to find a reasonable Lagrangian for these fields only. Instead, we add the vector fields $R_{a}$ (commuting; $U=-2$ ) and $\xi_{a}$ (anticommuting; $U=-1$ ), with

$$
\delta R_{a}=i \varepsilon \xi_{a}, \quad \delta \xi_{a}=\varepsilon C^{a} D_{\alpha} R_{a}-i \frac{\varepsilon}{2} \Lambda \varepsilon_{a b} R^{b}+\frac{k \varepsilon}{2} S R_{a}
$$

As in [18], there is no completely satisfactory choice for the conformal weight of $R_{a}$ and $\xi_{a}$, so for the moment we simply denote this as an unknown $k$. With these transformation laws one gets

$$
\left(\delta_{\eta} \delta_{\varepsilon}-\delta_{\varepsilon} \delta_{\eta}\right) \xi_{a}=-2 i \eta \varepsilon C^{\alpha} D_{\alpha} \xi_{a}+\eta \varepsilon \cdot \Lambda \varepsilon_{a b} \xi^{b}-i \eta \varepsilon k S \xi_{a},
$$

and the same equation with $\xi_{a} \rightarrow R_{a}$. (It is tedious to verify (4.9), but the form of (4.8) makes the corresponding equation for $R_{a}$ obvious.) It is important to point out that although in (4.8) and (4.9) we are considering fields $R$ and $\xi$ that transform in the vector representation of the Lorentz group, these formulas generalize immediately (with an obvious modification of the $\Lambda R$ term in (4.8)) to other representation. Thus, these formulas in fact describe the coupling to topological gravity of a multiplet with arbitrary Lorentz quantum numbers and conformal 
dimension. $R$ and $\xi$ have been chosen in a (not quite successful) attempt to write a simple invariant Lagrangian.

Since the transformation laws close without use of the equations of motion, using only conformal invariance, diffeomorphism invariance, and invariance under change of frame, there is a simple recipe for trying to find an invariant Lagrangian. Let $Z$ be any conformally invariant and generally covariant functional. Then $\mathscr{L}=\{Q, Z\}$ obeys $\{Q, \mathscr{L}\}=0$, since $\left\{Q^{2}, Z\right\}=0$. Unfortunately, with the minimal gravitational multiplet that we are considering, there is no completely satisfactory choice of $Z$. One would like to try

$$
Z_{0}=-\int d^{2} \sigma(\operatorname{det} e) \cdot e^{\alpha a} D_{\alpha} R^{b} \cdot \psi_{a b} .
$$

Here we meet a difficulty that arose in [18] (and was originally pointed out to me by $\mathrm{S}$. Axelrod). Equation (4.10) is invariant under global conformal transformations if and only if $R$ has conformal weight $k=1$, but in that case the integrand in (4.10) does not transform with any definite weight under local conformal transformations. The integrand in (4.10) has definite conformal weight under local conformal transformations only if $k=-1$, but in that case the conformal weight of the integrand is -2 (if we include the det $e$ factor), rather than 0 , so (4.10) is not locally conformally invariant. To save the day, one needs an additional Lorentz scalar field $\Phi$ of conformal weight 2 . In the presence of such a field, one can write

$$
Z=-\int d^{2} \sigma(\operatorname{det} e) \Phi \cdot e^{\alpha a} D_{\alpha} R^{b} \cdot \psi_{a b}
$$

This leads to the Lagrangian

$$
\begin{aligned}
\mathscr{L}= & \int d^{2} \sigma(\operatorname{det} e) \Phi\left[i \xi^{b} D^{a} \psi_{a b}+\frac{1}{2} R^{b} D^{a}\left(D_{a} C_{b}+D_{b} C_{a}-\delta_{a b} D_{f} C^{f}\right)\right. \\
& \left.-i R^{b}\left(2 \psi^{a c} D_{c} \psi_{a b}-\psi_{b c} D_{a} \psi^{a c}-\psi_{a c} D_{b} \psi^{a c}\right)+\cdots\right] .
\end{aligned}
$$

Here '...' refers to terms proportional to $D_{\alpha} \Phi$ and $\{Q, \Phi\}$. To complete the story, one must explain the construction of $\Phi$ in terms of suitable elementary degrees of freedom. Not having a particularly good proposal to make, I will not enter into this here.

4.1 Sigma Models Coupled to Topological Gravity. Now we would like to couple the sigma model of Sect. 2 to topological gravity. The inconclusive state of the discussion at the end of the last section will not matter, because we will in fact be coupling the sigma model of Sect. 2 to the minimal $e, \psi, C$ multiplet.

First of all, the fermionic transformation laws of Eq. (2.9) need to be modified. In the absence of gravity, we required $\left(\delta_{\eta} \delta_{\varepsilon}-\delta_{\varepsilon} \delta_{\eta}\right) \Psi=0$ for every $\Psi$. In the presence of gravity, the desired transformation laws (taking account of the Lorentz quantum numbers and conformal spin) are

$$
\begin{aligned}
\left(\delta_{\eta} \delta_{\varepsilon}-\delta_{\varepsilon} \delta_{\eta}\right) \chi^{i} & =-2 i \varepsilon \eta C^{\alpha} D_{\alpha} \chi^{i}, \\
\left(\delta_{\eta} \delta_{\varepsilon}-\delta_{\varepsilon} \delta_{\eta}\right) u^{i} & =-2 i \varepsilon \eta C^{\alpha} \partial_{\alpha} u^{i} \\
\left(\delta_{\eta} \delta_{\varepsilon}-\delta_{\varepsilon} \delta_{\eta}\right) \rho_{a}{ }^{i} & =-2 i \eta \varepsilon C^{\alpha} D_{\alpha} \rho_{a}{ }^{i}+\eta \varepsilon \Lambda \varepsilon_{a b} \rho^{b i}-i \eta \varepsilon S \rho_{a}^{i} \\
\left(\delta_{\eta} \delta_{\varepsilon}-\delta_{\varepsilon} \delta_{\eta}\right) H_{a}^{i} & =-2 i \eta \varepsilon C^{\alpha} D_{\alpha} H_{a}^{i}+\eta \varepsilon \Lambda \varepsilon_{a b} H^{b i}-i \eta \varepsilon S H_{a}^{i} .
\end{aligned}
$$


Clearly, our previous transformation laws will not do. The modified version

$$
\delta \chi^{i}=\varepsilon^{\alpha} C_{\alpha} u^{i}, \quad \delta u^{i}=i \varepsilon \chi^{i}
$$

may easily be seen to give the desired closure of the algebra on $u$ and $\chi$. Our previous transformation law for $\rho$ was

$$
\delta \rho_{a}{ }^{i}=H_{a}^{i}+\frac{i}{2} \varepsilon_{a b}\left(D_{k} J^{i}{ }_{j}\right) \chi^{k} \rho^{b_{J}}-i \varepsilon \Gamma_{j k}^{i} \chi^{j} \rho_{a}{ }^{k},
$$

and there is no useful way to modify it, since any modification could be absorbed in redefining $H$. At this point, we may proceed as in Sect. 2. The $H$ transformation law is uniquely determined by requiring that the algebra closes on $\rho$. This gives an even less promising-looking modification of (2.10):

$$
\begin{aligned}
\delta H_{a}^{i}= & -\frac{\varepsilon}{4} \chi^{k} \chi^{l}\left(R_{k l i t}+R_{k l^{\prime} t^{\prime}} J^{i^{\prime} i} J^{t^{\prime} t}\right) \rho_{a}^{t}+\frac{i \varepsilon}{2} \varepsilon_{b}^{a} \chi^{k} D_{k} J_{j}^{i} H^{b j}-\frac{\varepsilon}{4}\left(\chi^{k} D_{k} J^{i}\right)\left(\chi^{l} D_{l} J_{t}^{s}\right) \rho^{a t} \\
& -\frac{i \varepsilon}{2} \varepsilon_{a b}\left(C^{\alpha} \partial_{\alpha} u^{k}\right) D_{k} J_{j}^{i} \rho^{b j}+i \varepsilon\left(C^{b} D_{b} \rho_{a}^{i}+\frac{i}{2} \Lambda \varepsilon_{a b} \rho^{b i}+\frac{1}{2} S \rho_{a}^{i}\right)-i \varepsilon \Gamma_{j k}^{i} \chi^{j} \rho_{a}^{k} .
\end{aligned}
$$

Attractive or not, (4.16) can be seen to lead to closure of the algebra.

An invariant Lagrangian can now be found just as in Sect. 2. In other words, $\mathscr{L}=\{Q, V\}$, where now

$$
V=\int d^{2} \sigma \operatorname{det} e\left(\rho_{i}^{a} D_{a} u^{i}-\frac{1}{4} \rho_{i}^{a} H_{a}^{i}\right) .
$$

Upon evaluating $\{Q, V\}$, and eliminating $H$, one arrives at the generalization of (2.16):

$$
\begin{aligned}
\mathscr{L}= & \int d^{2} \sigma \operatorname{det} e\left[\frac{1}{2} g_{i j} \partial_{\alpha} u^{i} \partial^{\alpha} u^{j}+\frac{1}{2} \varepsilon^{\alpha \beta} J_{i j} \partial_{\alpha} u^{i} \partial_{\beta} u^{j}-i \rho^{a}{ }_{i}\left[D_{a} \chi^{i}+\frac{1}{2} \varepsilon_{a b}\left(\partial^{b} u^{j}\right) D_{k} J^{i}{ }_{j} \chi^{k}\right.\right. \\
& \left.-\psi_{a b} \partial^{b} u^{i}\right]-\frac{1}{8} \chi^{l} \chi^{k} R_{l k i t} \rho^{a i} \rho_{a}{ }^{t}-\frac{1}{16} \chi^{l} \chi^{k} \rho^{a}{ }_{i} \rho_{a t}\left(D_{l} J^{j i}\right)\left(D_{k} J_{j t}\right) \\
& \left.+\frac{i}{8} \varepsilon_{a b} \rho^{a i} \rho^{b}{ }_{i}\left(\varepsilon^{s t} D_{s} C_{t}-i \varepsilon^{s t} \psi_{s u} \psi_{t}{ }^{u}\right)+\frac{i}{4} \rho_{a}{ }^{i} C^{b} D_{b} \rho_{a}{ }^{i}\right]
\end{aligned}
$$

Equation (4.18) is automatically invariant under the fermionic symmetry, by virtue of the construction.

Now let us briefly discuss the properties of these models at the quantum level. The discussion will necessarily be provisional, because the proposal (4.12) for the gravitational action was incomplete (because of the lack of specification of $\Phi$ ). Nevertheless, I believe that the following remarks, in which we will naively ignore this problem and pretend that $\Phi=1$, are illuminating and may lead to insight about how the gravitational multiplet should be treated.

First of all, even in the conformal gauge (and even if we pretend $\Phi=1$ ), the action (4.12) of the purely gravitational multiplet does not become quadratic, because of the $R \psi D \psi$ "Yukawa" couplings. Nevertheless, the purely gravitational model is soluble quantum mechanically, because there are only finitely many Feynman diagrams contributing to any given correlation function. The reason for this is that if we define a quantum number $U_{B}$ that is 1 for $C,-1$ for $R$, and zero 
for all other fields, then the Yukawa couplings violate $U_{B}$ by -1 unit, and the rest of the Lagrangian is $U_{B}$ conserving. Therefore, any given correlation function with a given $U_{B}$ quantum number receives contributions only from diagrams with a definite number of $R \psi D \psi$ vertices.

Second, since in these models $Q^{2}=0$ only up to a conformal transformation, these models are consistent only if they are conformally invariant at the quantum level. Let us discuss this issue. We will consider only the case in which $M$ is flat (with the standard almost complex structure), so that it is only necessary to compute the central charge $c$ in the Virasoro algebra; we wish to know if this vanishes.

First of all, the anticommuting fields $\xi, \psi$ are just like the usual conformal ghosts $b, c$ of the bosonic string. Indeed, their kinetic operator is an operator $P$ mapping vectors to symmetric traceless tensors, with

$$
(P \xi)_{a b}=D_{a} \xi_{b}+D_{b} \xi_{a}-\delta_{a b} D_{f} \xi^{f} .
$$

This is the usual kinetic operator of the $b, c$ system. The $\xi, \psi$ Gaussian integral gives an effective action $\operatorname{det} P$ and contributes -26 to the Virasoro anomaly, ${ }^{10}$ this being the usual contribution of the conformal ghosts.

As for the bosons $R, C$, their kinetic operator is $P^{+} P$, so the effective action is $\operatorname{det}^{-1}\left(P^{+} P\right)=\operatorname{det}^{-2} P$, and the contribution to the anomaly is thus $+2.26=+52$.

Finally, in conformal gauge fixing of this system, we will have to introduce the conformal ghosts $b, c$, which will contribute -26 to the anomaly. The total is thus $-26+52-26=0$. Therefore, the purely gravitational model is quantum conformally invariant.

Now we consider the "matter" multiplet $u, \%, \rho$. Since the Lagrangian requires an almost complex structure, the basic multiplet includes two real bose fields $u^{1}$, $u^{2}$ (or a complex bose field), contributing +2 to the central charge. The right moving components of the basic multiplet are two real fermions, $\chi$ of spin 0 and $\rho$ of spin $1 .{ }^{11}$ It is well known that a pair of fermions of $\operatorname{spin} \frac{1}{2} \pm j$ contribute $1-12 j^{2}$ to the conformal anomaly. In the case at hand, $j=\frac{1}{2}$, and the contribution is -2 . This cancels the contribution +2 from the $u^{i}$. Thus, coupling to the free matter multiplet preserves the quantum conformal invariance.

Thus, if it is correct to interpret the models discussed in this paper in terms of an unbroken phase of string theory, we conclude that the restriction to $D=26$ or $D=10$ holds only in the broken phase, while $D$ is undetermined in the unbroken phase. Of course, the above reasoning is somewhat naive, because of the difficulty that has been noted in the formulation of (4.12). The challenge is really to reformulate the action of the gravitational multiplet to justify the above considerations.

Finally, let us briefly discuss the BRST invariant vertex operators than can be defined after coupling to two dimensional gravity. In Sect. 3, we defined, for every

\footnotetext{
10 In units in which a real boson contributes +1

11 The counting is as follows. In the basic multiplet, the two bosons $u^{i}, l=1,2$ are paired with $\chi^{l}, i=1,2$ and $\rho_{a}{ }^{l}, i=1$, 2. Since $\rho_{a}{ }^{l}=J^{i}{ }_{j} \varepsilon_{a b} \rho^{{ }^{b}}, \rho$ has two real components of spin \pm 1 , one of which is right moving and one left moving. Of the two components of $\chi^{i}$, one is right moving and one is left moving
} 
closed differential form $A$ on $M$, three BRST invariant operators $\mathcal{O}_{A}{ }^{(0)}, \mathcal{O}_{A}{ }^{(1)}$, and $\mathcal{O}_{A}{ }^{(2)}$. We must re-examine these because of the modified fermionic transformation law in (4.15). It can be seen that the $\mathcal{O}_{A}{ }^{(0)}$, for instance, are no longer BRST invariant, and the relation $\left\{Q, \mathcal{O}_{A}{ }^{(1)}\right\}=-i d \mathcal{O}_{A}{ }^{(0)}$ is likewise ruined. However, one still has

$$
d \mathcal{O}_{A}^{(1)}=i\left\{Q, \mathcal{O}_{A}{ }^{(2)}\right\}
$$

despite the correction to (4.15). Therefore, for any closed form $A$ on $M$,

$$
W_{A}(\Sigma)=\int_{\Sigma} \mathcal{O}_{A}^{(2)}
$$

is BRST invariant. So for any choice of closed forms $A_{1}, \ldots, A_{k}$, the correlation functions

$$
\left\langle\prod_{i=1}^{k} W_{A_{1}}(\Sigma)\right\rangle
$$

are topological invariants.

It may seen that this construction is less powerful than that in Sect. 3, for we seem to be defining fewer invariants. However, one may expect that in many interesting situations, the invariants of Sect. 3 will be trivial, but (4.22) non-trivial. For in Sect. 3, we considered an arbitrarily prescribed curve $\Sigma$ of genus $g$, and obtained invariants of holomorphic maps $\phi: \Sigma \rightarrow M$. These invariants did not depend on the complex structure of $\Sigma$, so they are zero if by perturbing that structure the holomorphic maps of $\Sigma$ to $M$ disappear. Thus, invariants of Sect. 3 are zero unless there is a holomorphic map of the universal curve of genus $g$ into $M$-a severe restriction if $g>0 .{ }^{12}$ But in (4.22), since we have coupled to two dimensional gravity, we are integrating over the moduli space of curves, and thus we are detecting all holomorphic curves of genus $g$ in $M$. Thus, (4.22) should be non-trivial in many interesting situations. But we leave the investigation of this for the future.

\section{Coupling to Gauge Fields}

In Sect. 2, we formulated a "BRST"-invariant generalization of the nonlinear sigma model, for maps $\Sigma \rightarrow M, \Sigma$ being a Riemann surface and $M$ an almost complex manifold. Then we generalized the space of maps $\Sigma \rightarrow M$ to the space of sections of an $M$-bundle $X$ over $\Sigma$

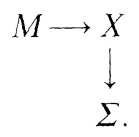

This generalization involved introducing gauge fields of the group $\operatorname{diff} M$ of diffeomorphisms of $M$. Those gauge fields - like the metric of $\Sigma$ - played a purely passive role in constructing the fermionic symmetry of Eq. (2.16). Indeed, the metric

12 An example for $g=1$ obeying this restriction was given at the end of Sect. 3 
and gauge field were regarded as part of the definition of the problem, and were invariant under the fermionic symmetry.

In Sect. 5, we generalized the construction of Sect. 2 to include coupling to dynamical two dimensional gravity, while preserving the fermionic symmetry. In the present section, we will do the analogue of this for gauge fields; we will couple the model of Sect. 2 to dynamical gauge fields in a BRST-invariant fashion.

In doing so, we will consider what is conceivably not the most general case. We will take the gauge group $G$ to be a compact group of automorphisms of $M$, leaving fixed a metric $g_{i j}$ and an almost complex structure $J^{i}{ }_{j}$.

The $G$ action on $M$ is generated by certain vector fields $V_{a}^{i}, a=1, \ldots, n$ ( $n$ being the dimension of $G$ ). The assertion that the $V_{a}^{i}$, for $a=1, \ldots, n$ leave fixed the metric $g_{i j}$ is equivalent to the assertion that they obey the Killing vector equation,

$$
D_{i} V_{j a}-D_{j} V_{i a}=0 \text {. }
$$

A useful consequence of this equation is

$$
D_{k} D_{s} V_{i a}=R_{\text {sitk }} V_{a}^{t} .
$$

The assertion that the almost complex structure is $G$-invariant means that the Lie derivatives $\mathscr{L}_{V_{a}}(J)$ are zero, or concretely that

$$
0=V_{a}^{k} D_{k} J^{i}{ }_{j}-J^{k}{ }_{j} D_{k} V_{a}^{i}+J^{i}{ }_{k} D_{j} V_{a}^{k} .
$$

Finally, the statement that the $V_{a}$ generate a $G$ action implies that

$$
\left[V_{a}, V_{b}\right]=f_{a b}{ }^{c} V_{c},
$$

with $f_{a b}{ }^{c}$ being the structure constants of $G$. The symbol $\left[V_{a}, V_{b}\right]$, denotes, of course, the commutator of the operators $V_{a}^{i}\left(\partial / \partial u^{i}\right)$,

$$
\left[V_{a}, V_{b}\right]^{j}=V_{a}^{i} \partial_{i} V_{b}^{j}-V_{b}^{i} \partial_{i} V_{a}^{j} .
$$

The first step in constructing a gauge invariant generalization of the sigma model of Eq. (2.16) is to pick a gauge multiplet. Here we may borrow from the results of [4]. In addition to the gauge field, which is a Lie algebra valued one form $A_{\alpha}{ }^{a}$, we introduce an anticommuting one form $\psi_{\alpha}^{a}$, and a commuting zero form $\phi^{a}$. The conformal dimension and ghost number of $(A, \psi, \phi)$ are

Table III. The basic gauge multiplet consists of $A, \phi$, and $\psi ; \hat{\lambda}, \eta$, and $\chi$ are needed to write a Lagrangian for those fields

\begin{tabular}{llrl}
\hline & $D$ & $U$ & Statistics \\
\hline$A_{\alpha}{ }^{a}$ & 1 & 0 & + \\
$\psi_{\alpha}{ }^{a}$ & 1 & 1 & - \\
$\phi^{a}$ & 0 & 2 & + \\
$\lambda^{a}$ & 2 & -2 & + \\
$\eta^{a}{ }^{a}$ & 1 & -1 & - \\
$\chi_{\alpha \beta}{ }^{a}$ & 1 & -1 & - \\
\hline
\end{tabular}


$(1,1,0)$, and $(0,1,2)$. As in $[4]$, the transformation laws are

$$
\delta A_{\alpha}=i \varepsilon \psi_{\alpha}, \quad \delta \psi_{\alpha}=-\varepsilon D_{\alpha} \phi, \quad \delta \phi=0 .
$$

One sees

$$
\begin{aligned}
\left(\delta_{\eta} \delta_{\varepsilon}-\delta_{\varepsilon} \delta_{\eta}\right) A_{\alpha} & =-D_{\alpha}(-2 i \eta \varepsilon \phi), \\
\left(\delta_{\eta} \delta_{\varepsilon}-\delta_{\varepsilon} \delta_{\eta}\right) \psi_{\alpha} & =\left[-2 i \eta \varepsilon \phi, \psi_{\alpha}\right], \\
\left(\delta_{\eta} \delta_{\varepsilon}-\delta_{\varepsilon} \delta_{\eta}\right) \phi & =0 .
\end{aligned}
$$

The right-hand side of (5.8) is an infinitesimal gauge transformation with gauge parameter $\Lambda=-2 i \eta \varepsilon \phi$.

To strictly follow the procedure of Sect. 5, we should now write an invariant kinetic energy for $(A, \psi, \phi)$. This requires introducing additional fields, indicated in Table III. $(A, \psi$, and $\phi$ are analogous to the minimal multiplet $e, \psi, C$ of Sect. 5; the additional fields needed to write a Lagrangian are the analogues of $R, \xi$.) We will temporarily postpone this step, and instead consider the coupling to the nonlinear sigma model, that is to the fields $u^{i}, \chi^{i}$, and $\rho_{\alpha}{ }^{i}$ of Sect. 2.

The fermionic transformation laws of Eq. (2.9) for the $u, \chi, \rho$ multiplet must be modified, because (2.9) leads to $\delta_{\eta} \delta_{\varepsilon}-\delta_{\varepsilon} \delta_{\eta}=0$, while in fact, according to (5.8), $\delta_{\eta} \delta_{\varepsilon}-\delta_{\varepsilon} \delta_{\eta}$ should be a gauge transformation. So we try

$$
\delta u^{i}=i \varepsilon \chi^{i}, \quad \delta \chi^{i}=\varepsilon \phi^{a} V_{a}^{i} .
$$

A similar modification of the transformation laws are made in Sect. 3, for similar reasons. Equation (5.9) leads as desired to

$$
\begin{aligned}
& \left(\delta_{\eta} \delta_{\varepsilon}-\delta_{\varepsilon} \delta_{\eta}\right) u^{i}=-2 i \eta \varepsilon \phi^{a} V_{a}^{i}, \\
& \left(\delta_{\eta} \delta_{\varepsilon}-\delta_{\varepsilon} \delta_{\eta}\right) \chi^{i}=-2 i \eta \varepsilon \phi^{a}\left(\partial_{j} V_{a}^{i}\right) \cdot \chi^{j} .
\end{aligned}
$$

Next, we need to determine the transformation laws of $\rho$ and $H$. As in Sect. 3, there is no useful way to modify the transformation law of $\rho$, which remains

$$
\delta \rho_{\alpha}{ }^{i}=\varepsilon H_{\alpha}{ }^{i}+\frac{i}{2} \varepsilon_{\alpha \beta}\left(D_{k} J_{l}^{i}\right) \chi^{k} \rho^{\beta l}-i \varepsilon \Gamma^{i}{ }_{k} \chi^{j} \rho_{\alpha}{ }^{k} .
$$

Any modification of (5.11) could be absorbed in a redefinition of $H$. It remains to determine the transformation law of $H$. Just as in Sects. 2 and 3, the transformation law of $H$ is uniquely determined by insisting that one should get the desired equation

$$
\left(\delta_{\eta} \delta_{\varepsilon}-\delta_{\varepsilon} \delta_{\eta}\right) \rho_{\alpha}^{i}=-2 i \eta \varepsilon \phi^{\alpha}\left(\partial_{j} V_{a}^{i}\right) \rho_{\alpha}^{j} .
$$

In this way, we arrive at the formula

$$
\begin{aligned}
\delta H_{\alpha}^{i}= & -\frac{\varepsilon}{4} \chi^{k} \chi^{l}\left(R_{k l t}{ }^{i}+R_{k l i^{\prime} t^{\prime}} J^{i \prime i} J^{t^{\prime} t}\right) \rho_{\alpha}^{t}+i \frac{\varepsilon}{2} \cdot \varepsilon^{\alpha}{ }_{\beta} \cdot \chi^{k} D_{k} J^{i}{ }_{j} H^{\beta j}-\frac{\varepsilon}{4}\left(\chi^{k} D_{k} J^{i}{ }_{s}\right)\left(\chi^{l} D_{l} J_{t}{ }^{s}\right) \rho_{\alpha}^{t} \\
& -i \frac{\varepsilon}{2} \phi^{a} V_{a}^{k}\left(D_{k} J^{i}{ }_{j}\right) \varepsilon_{\alpha \beta} \rho^{\beta j}+i \varepsilon \phi^{a}\left(D_{j} V_{a}^{i}\right) \rho_{\alpha}^{j}-i \varepsilon \Gamma_{j k}^{i} \chi^{j} H_{\alpha}^{k} .
\end{aligned}
$$

By calculations similar to those required in Sects. 2 and 3, it can be seen that (5.13) does lead to the right formula (just like (5.12), but with $H$ replacing $\rho$ ) for closing the algebra on $H$. Having in this way closed the algebra on all fields, it is now 
straightforward to find an invariant Lagrangian generalizing (2.16). It is simply $\mathscr{L}=\{Q, V\}$, where again

$$
V=\int d^{2} \sigma\left(\rho_{i}^{\alpha} D_{\alpha} u^{i}-\frac{1}{4} \rho_{l}^{\alpha} H_{\alpha}^{i}\right)
$$

Upon evaluating $\{Q, V\}$ and eliminating $H$, one finds this time the invariant generalization of (2.16),

$$
\begin{aligned}
\mathscr{L}_{M}= & \int d^{2} \sigma\left[\frac{1}{2} g_{i j} D_{\alpha} u^{i} D^{\alpha} u^{j}+\frac{1}{2} \varepsilon^{\alpha \beta} J_{i j} D_{\alpha} u^{i} D_{\beta} u^{j}-i \rho^{\alpha}{ }_{i}\left(D_{\alpha} \chi^{i}+\frac{1}{2} \varepsilon_{\alpha \beta}\left(D_{k} J^{i}{ }_{j}\right) \chi^{k} D^{\beta} u^{j}+\psi_{\alpha}{ }^{a} V_{a}{ }^{i}\right)\right. \\
& \left.+\frac{i}{4} \rho_{\alpha}{ }^{i} \rho^{\alpha s}\left(D_{s} V_{i a}\right) \phi^{a}-\frac{1}{8} \chi^{k} \chi^{l} \rho^{\alpha}{ }_{i} \rho_{\alpha t} R_{k l}{ }^{i t}-\frac{1}{16} \chi^{k} \chi^{l} \rho^{\alpha}{ }_{i} \rho_{\alpha}{ }^{t}\left(D_{k} J^{j i}\right)\left(D_{l} J_{j t}\right)\right]
\end{aligned}
$$

At this point, we would like to couple the "matter" action of Eq. (5.15) to an appropriate action of the gauge multiplet. The obvious idea is to formulate an appropriate version of two dimensional topological Yang-Mills theory (with BRST-like symmetry). After adding appropriate additional fields to the $(A, \psi, \phi)$ system, one could indeed find two dimensional gauge actions with BRST-like symmetry. ${ }^{13}$ This is, however, not what we will do here. We will go in another direction, which may seem less obvious at first sight.

One of the intriguing ideas in [3] involved the Jones polynomial [6] of knot theory. In fact, the Jones polynomial is mysterious because, though one would wish to understand it in three dimensional terms (since it describes a knot in three-space), no intrinsic three dimensional description of this invariant is known.

Atiyah proposed in [3] that a natural (intrinsically three dimensional) understanding of the Jones polynomial should be found by relating it to Floer and Donaldson theory. This proposal was accompanied by a whole list of analogies between Floer theory and the Jones polynomial. If this philosophy is valid, one would ideally like to implement it in the context of a relativistic quantum field theory. The following remarks, aiming toward a way of doing this, can be seen as an attempt to sharpen Atiyah's conjecture.

A knot is an embedding of a circle $S$ in a three manifold $Y^{14}$. Let us think physically $-Y$ is physical three dimensional space and $S$ might be a superstring or cosmic string in $Y$. When time dependence is included, the knot $S$ should be replaced by its world sheet, which is a Riemann surface $\Sigma$. And $Y$ is replaced by space-time, which is a four manifold $M$. Thus, to work relativistically, we should study not a knot on a three manifold, but a Riemann surface $\Sigma$ imbedded in a smooth four manifold $Z$. From this point of view, "knot theory" refers to the case that $\Sigma$ is $S \times R^{1}$ and $Z$ is $Y \times R^{1}$, with $R^{1}$ representing "time" and $S$ a knot in the three manifold $Y$. We wish to formulate a relativistic quantum field theory suitable for studying an embedding $\phi: \Sigma \rightarrow Z^{15}$.

\footnotetext{
13 One way to do this (though it presumably does not give the minimal two dimensional model) is dimensional reduction of the model of [4] from four to two dimensions

${ }^{14} \mathrm{Up}$ to the present, the Jones polynomial has been defined only for $Y=S^{3}$. If the present discussion is on the right track, the Jones polynomial generalizes to other $Y$

15 We regard the embedding as given and fixed; we will not include dynamical degrees of freedom representing the map $\phi: \Sigma \rightarrow Z$
} 
To do this, we will simply couple a nonlinear sigma model on $\Sigma$ to a gauge theory on $Z$. So we pick a compact gauge group $G$, and write down a four dimensional gauge theory on $Z$ with BRST-like symmetry. This requires [4], along with the minimal gauge multiplet $(A, \psi, \phi)$, some additional fields $\eta, \lambda, \chi_{\alpha \beta}$ as summarized in Table III. From [4], the Lagrangian is

$$
\begin{aligned}
\mathscr{L}_{G}= & \int_{Z} \operatorname{Tr}\left[\frac{1}{4} F_{\alpha \beta} F^{\alpha \beta}+\frac{1}{2} \phi D_{\alpha} D^{\alpha} \lambda-i \eta D_{\alpha} \psi^{\alpha}+i D_{\alpha} \psi_{\beta} \cdot \chi^{\alpha \beta}\right. \\
& \left.-\frac{i}{8} \phi\left[\chi_{\alpha \beta}, \chi^{\alpha \beta}\right]-\frac{i}{2} \lambda\left[\psi_{\alpha}, \psi^{\alpha}\right]\right] .
\end{aligned}
$$

The fermionic transformation laws from [4] are

$$
\begin{aligned}
\delta A_{\alpha} & =i \varepsilon \psi_{\alpha}, \quad \delta \psi_{\alpha}=-\varepsilon D_{\alpha} \phi, \quad \delta \phi=0, \quad \delta \eta=\frac{1}{2} \varepsilon[\phi, \lambda], \\
\delta \lambda & =2 i \varepsilon \eta, \quad \delta \chi_{\alpha \beta}=\varepsilon\left(F_{\alpha \beta}+\frac{1}{2} \varepsilon_{\alpha \beta \gamma \delta} F^{\gamma \delta}\right) .
\end{aligned}
$$

Note that the first three of these equations coincide with (5.7). Therefore, if we simply forget about $\eta, \lambda, \chi$ and restrict the zero form $\phi$ and the one forms $A$ and $\psi$ to a Riemann surface $\Sigma$ embedded in $Z$, we get a collection of fields that transform just like the two dimensional gauge multiplet.

Now, let $M$ be an almost complex manifold with $G$ symmetry. Introduce fields $u^{i}, \chi^{i}, \rho_{\alpha}{ }^{i}$ describing a nonlinear sigma model of maps $\Sigma \rightarrow M$. This system can be made gauge invariant as well as BRST-invariant by coupling to $A, \phi, \lambda$ as in (5.15). The combined Lagrangian

$$
\mathscr{L}=\mathscr{L}_{M}+\mathscr{L}_{G}
$$

is then invariant under the transformation described in (5.11) and (5.17). It is suitable for studying topological invariants associated with a four manifold $Z$ and an embedded Riemann surface $\Sigma$. By specializing to the case $Z=Y \times R^{1}$, $\Sigma=S \times R^{1},(5.18)$ can be specialized to knot theory.

One can hope to find interesting topological invariants of the pair $(Z, \Sigma)$ in the form

$$
\left\langle\prod_{i=1}^{k} \mathcal{O}_{i}\right\rangle,
$$

with the $\mathcal{O}_{i}$ being non-trivial BRST invariant operators, that is, operators that are not of the form $\{Q, \Lambda\}$ for any $\Lambda$. We will describe briefly what operators of this form exist, but we will not try here to evaluate their expectation values.

First of all, we have operators that are constructed only from the four dimensional system $(A, \psi, \phi, \eta, \lambda, \chi)$. To construct them, one begins with $W_{0}=$ $\frac{1}{2} \operatorname{Tr} \phi^{2}$, and then solves the equations

$$
\begin{aligned}
& d W_{0}=i\left\{Q, W_{1}\right\}, \quad d W_{1}=i\left\{Q, W_{2}\right\}, \quad d W_{2}=i\left\{Q, W_{3}\right\}, \\
& d W_{3}=i\left\{Q, W_{4}\right\}, \quad d W_{4}=0 .
\end{aligned}
$$

The resulting $W_{k}$ were described in [4]. Equation (5.20) implies that for any $k$ dimensional homology cycle $\gamma$ in $Z$,

$$
I(\gamma)=\int_{\gamma} W_{k}
$$


is BRST invariant and (up to a BRST commutator) depends only on the homology class of $\gamma$. The $I(\gamma)$ are quite non-trivial. In the absence of $\Sigma$, correlation functions of the $I(\gamma)$ are precisely the Donaldson invariants. In the presence of the $\Sigma$, these correlation functions may well receive new contributions.

We can also look for non-trivial BRST invariant operators constructed from the fields $u^{i}, \chi^{i}, \rho_{\alpha}{ }^{i}$ that are defined only on $\Sigma$. Recall that in Sect. 2, we defined an operator $\mathcal{O}_{A}{ }^{(0)}$ for every closed $n$ form $A$ on $M$ :

$$
\mathcal{O}_{A}{ }^{(0)}=A_{i_{1} l_{2}} i_{n}\left(u^{k}\right) \cdot \chi^{i_{1}} \chi^{i_{2}} \cdots \chi^{i_{n}} .
$$

In Sect. $2, \mathcal{O}_{A}{ }^{(0)}$ was BRST invariant if $d A=0$, and a BRST commutator if $A=d B$. But with the modified transformation laws, it is no longer true that $\mathcal{O}_{A}{ }^{(0)}$ is BRST invariant, even if $d A=0$. One finds

$$
\delta \mathcal{O}_{A}{ }^{(0)}=\varepsilon \cdot n \phi^{a} V_{a}^{i_{1}} A_{i_{1} i_{2} \cdots i_{n}} \chi^{i_{2}} \chi^{i_{3}} \cdots \chi^{i_{n}} .
$$

Can the situation by repaired by adding corrections to the definition of $\mathcal{O}_{A}{ }^{(0)}$ ? Let us first make a few useful preliminary observations. Let us introduce an operator $i_{a}$ of "contraction with $V_{a}$ " defined by saying that if $B$ is an $n$-form, then $i_{a}(B)$ is the $(n-1)$ form $\left(i_{a}(B)\right)_{i_{2} i_{3} \cdots i_{n}}=n V_{a}^{i_{1}} B_{i_{1} i_{2} \cdots i_{n}}$. Obviously, (5.23) can be written

$$
\delta \mathcal{O}_{A}{ }^{(0)}=\varepsilon \phi^{a} \mathcal{O}_{i_{a}}(A) .
$$

Now, let $\mathscr{L}_{a}$ be the Lie derivative with respect to the vector field $V_{a}$. The formula for the action of $\mathscr{L}_{a}$ on differential forms is

$$
\mathscr{L}_{a}(B)=\left(d i_{a}+i_{a} d\right) B .
$$

Therefore, if $B$ is closed, and in addition is $V_{a}$-invariant (so $\mathscr{L}_{a}(B)=0$ ), then

$$
d\left(i_{a}(B)\right)=0 .
$$

Now in (5.22), we may as well suppose that $\mathscr{L}_{a}(A)=0$, since by averaging over the compact group $G$, we can take $A$ to be $G$-invariant without changing its cohomology class. As we are also taking $d A=0$, we have according to (5.26),

$$
d\left(i_{a}(A)\right)=0 .
$$

Now, there may or may not exist an $n-2$ form $A_{a}$ such that

$$
i_{a}(A)=d A_{a} .
$$

If $A_{a}$ exists, we can try to "improve" the definition of $\mathcal{O}_{A}{ }^{(0)}$ to

$$
\tilde{\mathcal{O}}_{A}{ }^{(0)}=A_{i_{1} i_{2} \cdots i_{n}} \chi^{i_{1}} \chi^{i_{2}} \cdots \chi^{i_{n}}-\phi^{a} A_{a i_{1} i_{2} \cdots i_{n-2}} \chi^{i_{1}} \chi^{i_{2}} \cdots \chi^{i_{n-2}} .
$$

Now one computes that the BRST variation of $\tilde{\mathcal{O}}_{A}{ }^{(0)}$ is

$$
\delta \tilde{\mathbb{O}}_{A}{ }^{(0)}=-(n-2) \phi^{a} \phi^{b} V_{b}^{i_{1}} A_{a i_{1} i_{2} \cdots i_{n-2}} \chi^{i_{2}} \cdots \chi^{i_{n-2}} .
$$

In a very precise sense, (5.30) represents progress. In (5.23) the error was of $(n-1)^{s t}$ - order in $\chi$, but in $(5.30)$ it is of order $(n-3)$. Continuing in this way, adding additional terms of lower order in $\chi$, one after a finite number of steps either will succeed in finding a BRST invariant extension of $\mathcal{O}_{A}$ or will find an obstruction to this. 
The whole process can be described succinctly as follows. Let $\Omega *(M)$ be the de Rham complex of $M$, graded by dimension in the usual way. Let $S^{*}\left(\phi^{a}\right)$ be a polynomial algebra on the $\phi^{a}$, graded by considering them to be of degree two. Let $\Omega_{G}{ }^{*}(M)=\Omega *(M) \otimes S^{*}\left(\phi^{a}\right)$ (with the grading induced from that of the factors), and let $W^{*}$ be the $G$-invariant subcomplex of $\Omega_{G}{ }^{*}(M)$. An element of $W^{*}$ is an element of $\Omega_{G}{ }^{*}(M)$ that is annihilated by

$$
L_{a}=\mathscr{L}_{a}+f_{a b}{ }^{c} \phi^{b} \frac{\hat{\partial}}{\partial \phi^{c}},
$$

where $f_{a b}{ }^{c}=-f_{b a}{ }^{c}$ are the structure constants of $G$. Note that $\phi^{a} L_{a}=\phi^{a} \mathscr{L}_{a}$. Therefore, $W^{*}$ is annihilated by $\phi^{a} \mathscr{L}_{a}$,

$$
\phi^{a} \mathscr{L}_{a} \cdot W^{*}=0
$$

Now, let

$$
D=d+\phi^{a} i_{a} .
$$

$D$ is an operator on $\Omega_{G}{ }^{*}(M)$ of degree one. One computes

$$
D^{2}=\phi^{a} \mathscr{L}_{a} .
$$

So, using (5.32), $D^{2}=0$ on $W^{*}$.

Let $B$ be an element of $W^{*}$ of degree $n$. Any such $B$ has an expansion

$$
B=B^{(n)}+\phi^{a} B_{a}^{(n-2)}+\phi^{a} \phi^{b} B_{a b}^{(n-4)}+\cdots,
$$

where, for $k=n, n-2, n-4, \ldots, B^{(k)}$ is a $k$ form on $M$. Let

$$
\widehat{\mathcal{O}}_{B}^{(0)}=\mathcal{O}_{B^{(n)}}^{(0)}+\phi^{a} \mathcal{C}_{B_{a}^{(n-2)}}^{(0)}+\phi^{a} \phi^{b} \mathcal{O}_{B_{a b}(0)}^{(0)}{ }_{(n)}+\cdots,
$$

where (as before) for any differential form $A, \mathcal{O}_{A}{ }^{(0)}=A_{i_{1} i_{2} \cdots i_{k}}^{(k)} \chi^{i_{1}} \chi^{i_{2}} \cdots \chi^{i_{k}}$. The BRST transformation law can now be stated very simply. One finds

$$
\delta \widehat{O}_{B}^{(0)}=i \varepsilon \widehat{O}_{D B}{ }^{(0)} \text {. }
$$

Therefore, it is clear what is going on. The operator $\widehat{O}_{B}^{(0)}$ is BRST invariant if $D B=0$, and is a BRST commutator if $B=D \Lambda$ for some $\Lambda \in W^{*}$. The non-trivial BRST-invariant operators that can be constructed this way thus correspond exactly to the $D$-cohomology. According to $[19,20]$, this is the $G$-equivalent cohomology of $M$.

Just as in our previous discussion, one can push further, and define operators $\widehat{\mathcal{C}_{B}}{ }^{(1)}, \widehat{\mathcal{O}}_{B}^{(2)}$ obeying

$$
d \widehat{O}_{B}^{(0)}=i\left\{Q, \widehat{O}_{B}^{(1)}\right\}, \quad d \widehat{O}_{B}^{(1)}=i\left\{Q, \widehat{O}_{B}^{(2)}\right\} .
$$

The formulas are similar to the foregoing but a bit more elaborate. Equation (5.38) implies that for any $k$ cycle $\gamma$ on $\Sigma$, and any $D$-cohomology class $B$,

$$
J(\gamma, B)=\int_{\gamma} \widehat{O}_{B}^{(k)}
$$

is BRST-invariant. Correlation functions

$$
\left\langle\prod_{i=1}^{n} I\left(\gamma_{i}\right) \prod_{j=1}^{m} J\left(\gamma_{j}, B_{j}\right)\right\rangle
$$


(with the $I(\gamma)$ as defined in (5.21)) are intriguing invariants of the pair $(Z, \Sigma)$, and may or may not prove to shed light on the Jones polynomial.

Acknowledgements. The present work originated as a result of the influence of M. F. Atiyah, to whom I am greatly indebted. He anticipated many features of these constructions. I also benefitted from discussions with P. Kronheimer and S. Axelrod.

\section{References}

1. Donaldson, S.: An application of gauge theory to the topology of four manifolds. J. Differ. Geom. 18269 (1983); The orientation of Yang-Mills moduli spaces and 4-manifold topology. J. Differ Geom. 26397 (1987); Polynomial Invariants of Smooth Four-Manifolds, Oxford preprint

2. Floer, A.: An instanton invariant for three manifolds. Courant Institute preprint (1987); Morse theory for fixed points of symplectic diffeomorphisms. Bull. Am. Math. Soc. 16279 (1987)

3. Atiyah, M. F.: New invariants of three and four dimensional manifolds. To appear in the procecdings of the Symposium on the Mathematical Heritage of Hermann Weyl (Chapel Hill, May, 1987), ed. R. Wells et. al.

4. Witten, E.: Topological quantum field theory. Commun. Math. Phys. (in press)

5. Gromov, M.: Pseudo holomorphic curves in symplectıc manıfolds. Invent. Math. 82307 (1985); and in the Proceedings of the International Congress of Mathematicians, Berkeley (August, 1986), p. 81

6. Jones, V.: A polynomial invariant for knots via von neumann algebras. Bull. Am. Math. Soc. 82 $103(1985)$

7. Witten, W.: Superconducting strings. Nucl. Phys. B249 557 (1985)

8. Gross, D. J., Harvey, J. A., Martinec, E., Rohm. R.: Heterotic string theory. Nucl. Phys. B256 253 (1985)

9. Witten, E.: Cosmic superstrings. Phys. Lett. 153B 243 (1985)

10. Witten, E.: Topological gravity. IAS preprint (February 1988)

11. Horowitz, G. T., Lykken, J., Rohm, R., Strominger, A.: Phys. Rev. Lett. 57162 (1978)

12. Strominger, A.: Lectures on closed string field theory. To appear in the proceedings of the ICTP spring workshop, 1987

13. Gross, D. J.: High energy symmetries of string theory, Princeton preprint 1988

14. Friedan, D., Martinec, E., Shenker, S.: Nucl. Phys. B271 93 (1986)

15. Peskin, M.: Introduction to string and superstring theory. SLAC-PUB-4251 (1987)

16. Atiyah, M. F.: Concluding remarks at the Schloss Ringberg meeting (March, 1987)

17. Hooft, G. ' $t$ : Computation of the quantum effects due to a four dimensional pseudoparticle. Phys. Rev. D14 3432 (1976)

18. Witten, E.: Topological gravity: IAS preprint, February, 1988

19. Atiyah, M. F., Bott, R.: The moment map and equivariant cohomology. Topology 231 (1984)

20. Mathai, V., Quillen, D.: Superconnections, thom classes, and equivariant differential forms: Topology 25A 85 (1986)

Communicated by A. Jaffe

Received March 7, 1988 
